\title{
A data compendium of Mycobacterium tuberculosis antibiotic resistance
}

Alice Brankin ${ }^{4,},{ }^{* *}$ and Kerri M Malone ${ }^{19,{ }^{*}, * *}$, Ivan Barilar ${ }^{29}$, Simone Battaglia ${ }^{1}$, Emanuele Borroni $^{1}$, Angela Pires Brandao ${ }^{2,3}$, Andrea Maurizio Cabibbe ${ }^{1}$, Joshua Carter ${ }^{5}$, Daniela Maria Cirillo ${ }^{1}$, Pauline Claxton ${ }^{6}$, David A Clifton ${ }^{4}$, Ted Cohen ${ }^{7}$, Jorge Coronel ${ }^{8}$, Derrick W Crook ${ }^{4}$, Viola Dreyer $^{29}$, Sarah G Earle ${ }^{4}$, Vincent Escuyer ${ }^{9}$, Lucilaine Ferrazoli ${ }^{3}$, Philip W Fowler ${ }^{4}$, George Fu $\mathrm{Gao}^{10}$, Jennifer Gardy ${ }^{11}$, Saheer Gharbia ${ }^{12}$, Kelen Teixeira Ghisi ${ }^{3}$, Arash Ghodousi ${ }^{1,13}$, Ana Luíza Gibertoni Cruz ${ }^{4}$, Louis Grandjean ${ }^{33}$, Clara Grazian ${ }^{14}$, Ramona Groenheit ${ }^{44}$, Jennifer L Guthrie ${ }^{15,16}$, Wencong He ${ }^{10}$, Harald Hoffmann ${ }^{17,18}$, Sarah J Hoosdally ${ }^{4}$, Martin Hunt ${ }^{19,4}$, Zamin Iqbal ${ }^{19}$, Nazir Ahmed Ismail ${ }^{20}$, Lisa Jarrett ${ }^{21}$, Lavania Joseph $^{20}$, Ruwen Jou ${ }^{22}$, Priti Kambli ${ }^{23}$, Rukhsar Khot ${ }^{23}$, Jeff Knaggs $^{19,4}$, Anastasia Koch ${ }^{24}$, Donna Kohlerschmidt ${ }^{9}$, Samaneh Kouchaki $^{4,25}$, Alexander S Lachapelle ${ }^{4}$, Ajit Lalvani ${ }^{26}$, Simon Grandjean Lapierre ${ }^{27}$, Ian F Laurenson $^{6}$, Brice Letcher ${ }^{19}$, Wan-Hsuan Lin ${ }^{22}$, Chunfa Liu ${ }^{10}$, Dongxin Liu ${ }^{10}$, Ayan Mandal ${ }^{28}$, Mikael Mansjö ${ }^{44}$, Daniela Matias ${ }^{21}$, Graeme Meintjes $^{24}$, Flávia de Freitas Mendes ${ }^{3}$, Matthias Merker $^{29}$, Marina Mihalic ${ }^{18}$, James Millard ${ }^{30}$, Paolo Miotto ${ }^{1}$, Nerges Mistry ${ }^{28}$, David Moore ${ }^{31,8}$, Kimberlee A Musser ${ }^{9}$, Dumisani Ngcamu ${ }^{20}$, Hoang Ngoc Nhung ${ }^{32}$, Stefan Niemann ${ }^{29,} 48$, Kayzad Soli Nilgiriwala ${ }^{28}$, Camus Nimmo $^{33}$, Nana Okozi ${ }^{20}$, Rosangela Siqueira Oliveira ${ }^{3}$, Shaheed Vally $\mathrm{Omar}^{20}{ }^{2}$ Nicholas Paton ${ }^{34}$, Timothy EA Peto ${ }^{4}$, Juliana Maira Watanabe Pinhata ${ }^{3}$, Sara Plesnik ${ }^{18}$, Zully M Puyen ${ }^{35}$, Marie Sylvianne Rabodoarivelo ${ }^{36}$, Niaina Rakotosamimanana ${ }^{36}$, Paola MV Rancoita $^{13}$, Priti Rathod ${ }^{21}$, Esther Robinson ${ }^{21}$, Gillian Rodger ${ }^{4}$, Camilla Rodrigues ${ }^{23}$, Timothy C Rodwell ${ }^{37,38}$, Aysha Roohi ${ }^{4}$, David Santos-Lazaro ${ }^{35}$, Sanchi Shah ${ }^{28}$, Thomas Andreas Kohl ${ }^{29}$, Grace Smith $^{21,12}$, Walter Solano ${ }^{8}$, Andrea Spitaleri ${ }^{1,13}$, Philip Supply ${ }^{39}$, Utkarsha Surve ${ }^{23}$, Sabira Tahseen $^{40}$, Nguyen Thuy Thuong Thuong ${ }^{32}$, Guy Thwaites ${ }^{32,4}$, Katharina Todt ${ }^{18}$, Alberto 
25 Trovato $^{1}$, Christian Utpatel ${ }^{29}$, Annelies Van Rie ${ }^{41}$, Srinivasan Vijay ${ }^{42}$, Timothy M Walker ${ }^{4,32}$, A

26 Sarah Walker ${ }^{4}$, Robin Warren ${ }^{43}$, Jim Werngren ${ }^{44}$, Maria Wijkander ${ }^{44}$, Robert J Wilkinson ${ }^{45,46,26}$,

27 Daniel J Wilson ${ }^{4}$, Penelope Wintringer ${ }^{19}$, Yu-Xin Xiao ${ }^{22}$, Yang Yang ${ }^{4}$, Zhao Yanlin ${ }^{10}$, Shen-Yuan

$28 \mathrm{Yao}^{20}$, Baoli Zhu ${ }^{47}$

30 Institutions

$31 \quad{ }^{1}$ IRCCS San Raffaele Scientific Institute, Milan, Italy

$32{ }^{2}$ Oswaldo Cruz Foundation, Rio de Janeiro, Brazil

$33{ }^{3}$ Institute Adolfo Lutz, São Paulo, Brazil

$34{ }^{4}$ University of Oxford, Oxford, UK

$35{ }^{5}$ Stanford University School of Medicine, Stanford, USA

$36 \quad{ }^{6}$ Scottish Mycobacteria Reference Laboratory, Edinburgh, UK

$37{ }^{7}$ Yale School of Public Health, Yale, USA

$38 \quad 8$ Universidad Peruana Cayetano Heredia, Lima, Perú

$39{ }^{9}$ Wadsworth Center, New York State Department of Health, Albany, USA

$40 \quad{ }^{10}$ Chinese Center for Disease Control and Prevention, Beijing, China

$41{ }^{11}$ Bill \& Melinda Gates Foundation, Seattle, USA

$42 \quad 12$ UK Health Security Agency, London, UK

$43{ }^{13}$ Vita-Salute San Raffaele University, Milan, Italy

$44 \quad{ }^{14}$ University of New South Wales, Sydney, Australia

$45 \quad{ }^{15}$ The University of British Columbia, Vancouver, Canada

$46 \quad 16$ Public Health Ontario, Toronto, Canada

$47 \quad 17$ SYNLAB Gauting, Munich, Germany

$48{ }^{18}$ Institute of Microbiology and Laboratory Medicine, IMLred, WHO-SRL Gauting, Germany 
${ }^{19}$ EMBL-EBI, Hinxton, UK

$50 \quad{ }^{20}$ National Institute for Communicable Diseases, Johannesburg, South Africa

$51 \quad{ }^{21}$ Public Health England, Birmingham, UK

22 Taiwan Centers for Disease Control, Taipei, Taiwan

$53 \quad{ }^{23}$ Hinduja Hospital, Mumbai, India

${ }^{24}$ University of Cape Town, Cape Town, South Africa

${ }^{25}$ University of Surrey, Guildford, UK

${ }^{26}$ Imperial College, London, UK

27 Université de Montréal, Canada

${ }^{28}$ The Foundation for Medical Research, Mumbai, India

${ }^{29}$ Research Center Borstel, Borstel, Germany

60

${ }^{30}$ Africa Health Research Institute, Durban, South Africa

${ }^{32}$ Oxford University Clinical Research Unit, Ho Chi Minh City, Viet Nam

33 University College London, London, UK

${ }^{34}$ National University of Singapore, Singapore

35 Instituto Nacional de Salud, Lima, Perú

${ }^{41}$ University of Antwerp, Antwerp, Belgium 
${ }^{43}$ Stellenbosch University, Cape Town, South Africa

${ }^{44}$ Public Health Agency of Sweden, Solna, Sweden

${ }^{45}$ Wellcome Centre for Infectious Diseases Research in Africa, Cape Town, South Africa

${ }^{46}$ Francis Crick Institute, London, UK

${ }^{47}$ Institute of Microbiology, Chinese Academy of Sciences, Beijing, China

${ }^{48}$ German Center for Infection Research (DZIF), Hamburg-Lübeck-Borstel-Riems, Germany

*equal contribution authors

$81 \quad{ }^{* *}$ co-corresponding authors

\section{Abstract}

Consortium (CRyPTIC) presents here a global collection of 15,211 Mycobacterium tuberculosis clinical isolates, all of which have undergone whole genome sequencing and have had their minimum inhibitory concentrations to 13 antitubercular drugs measured. The isolates represent five major $M$. tuberculosis lineages originating from 23 countries across four continents. 6,814 isolates were found resistant to at least one drug, including 2,129 samples fully satisfy the clinical definitions of RR/MDR, pre-XDR or XDR. Resistance status to eight antitubercular drugs can be accurately predicted using a genetic mutation catalogue for over $90 \%$ of the isolates. Furthermore, we show the presence of suspected resistance conferring mutations for isolates resistant to the newly introduced drugs bedaquiline, clofazimine, delamanid and linezolid. Finally, a case study of rifampicin mono-resistance is presented to showcase how this compendium could be used to advance our genetic understanding of rare resistance 
phenotypes and evaluate the likely performance of a widely used molecular diagnostic

101 to come.

\section{Introduction}

Tuberculosis (TB) is a curable and preventable disease; $85 \%$ of those afflicted can be successfully treated with a six-month regimen. Despite this, TB is the world's top infectious disease killer with 10 million new cases and 1.2 million deaths estimated in 2019 alone [1]. Furthermore, drug resistant TB (DR-TB) is a continual threat; almost half a million people were estimated to have developed resistance to the first-line drug rifampicin (RR-TB), with three quarters of these cases estimated to be multidrug-

110 resistant (MDR-TB, resistant to first-line drugs isoniazid and rifampicin) [1]. Worryingly, only $44 \%$ of DR-TB cases were officially notified and just over half of these cases were successfully treated $(57 \%)[1]$.

To address these issues, the World Health Organisation (WHO) is encouraging

115 the development of better, faster and more targeted diagnostic and treatment

116 strategies through its EndTB campaign. Of particular interest is universal drug

117 susceptibility testing (DST). Conventionally, DST relies on lengthy (4 weeks minimum)

118 culture-based methods that require strict biosafety conditions for Mycobacterium

119 tuberculosis. The development of rapid genetics-based assays has decreased

120 diagnostic time to as little as 2 hours through the detection of specific resistance

121 conferring mutations e.g. the Cepheid Xpert@ MTB/RIF test [2, 3]. However, assay

122 bias towards specific genic regions can result in misdiagnosis of resistance, the 
123 prescription of ineffective treatment regimens and subsequent spread of multi-drug

124 resistant disease, as seen during an outbreak in Eswatini of an MDR strain harbouring an $r p o B$ I491F mutation not detected by the Xpert® MTB/RIF assay [4-6].

Furthermore, detection of rifampicin resistance with the Xpert@ MTB/RIF line probe assay (LPA) assay is used to infer MDR-TB as epidemiologically, rifampicin resistance tends to coincide with resistance to isoniazid [7]. While this modus operandi is successful at pragmatically identifying potential MDR cases quickly and effectively,

131 it is not generally true that a single path towards developing MDR or extensively drug

132 resistant TB $(\mathrm{XDR}=\mathrm{MDR} / \mathrm{RR}+$ resistance to at least one fluoroquinolone and either 133 bedaquiline or linezolid) exists. For example, M. tuberculosis strains that are sensitive

134 to rifampicin but resistant to multiple other anti-tubercular drugs would be missed by current LPA diagnostic testing and remain undetected in the community. A better 136 understanding of how various combinations of drug resistance in different

137 backgrounds (nonMDR/MDR) arises is essential to not only prevent the spread of resistant strains and to promote the use of effective treatment regimens, but also to

139 facilitate the development of suitable diagnostic assays unbiased in their approaches 140 to detect any and all resistance where it occurs.

Whole-genome sequencing (WGS) has the potential to reveal the entirety of

143 the $M$. tuberculosis genetic resistance landscape for any number of drugs

144 simultaneously whilst enabling a more rapid turnaround time and affordable cost in

145 comparison to DST culture methods [8]. The success of WGS as a diagnostic method

146 wholly depends on a comprehensive and accurate catalogue of resistance-conferring

147 mutations for each drug. Recent advances have shown that genotypic predictions of 
148 resistance correlate well with DST results for first-line drugs [7]. However, the

149 mechanisms of resistance to second-line, new and re-purposed drugs (NRDs) are less

150 well understood despite their increased administration in clinics as MDR cases climb

151 [9]. To align with the goals of the EndTB campaign, further research is vital to construct

152 an extensive catalogue of genetic variants associated with resistance, and indeed susceptibility, if WGS-based diagnostics are to become routine practice for DR-TB management. Consortium (CRyPTIC) has collected M. tuberculosis clinical isolates worldwide to survey the genetic variation associated with resistance to 13 antitubercular drugs, specifically the first-line drugs rifampicin, isoniazid, ethambutol, the second-line drugs amikacin, kanamycin, rifabutin, levofloxacin, moxifloxacin, ethionamide, and the new and re-purposed drugs bedaquiline, clofazimine, delamanid and linezolid. Here, we present a data compendium of 15,211 global clinical isolates, each of which has had

163 its genomic sequence determined and DST profile measured [10]. This compendium

164 is the largest drug screening effort to date for M. tuberculosis in a 'one isolate - one microscale assay' format across defined compound concentration ranges. Finally, this

166 matched genotypic-phenotypic dataset will prove an invaluable resource to accelerate

167 and improve AMR diagnostic development for TB, both by the enrichment of mutation catalogues for WGS resistance prediction and the identification of important diagnostic gaps and drug resistance patterns. 
173 Approval for CRyPTIC study was obtained by Taiwan Centers for Disease Control IRB

174 No. 106209, University of KwaZulu Natal Biomedical Research Ethics Committee

175 (UKZN BREC) (reference BE022/13) and University of Liverpool Central University

176 Research Ethics Committees (reference 2286), Institutional Research Ethics

177 Committee (IREC) of The Foundation for Medical Research, Mumbai (Ref nos.

$178 \mathrm{FMR} / \mathrm{IEC} / \mathrm{TB} / 01 \mathrm{a} / 2015$ and FMR/IEC/TB/01b/2015), Institutional Review Board of

179 P.D. Hinduja Hospital and Medical Research Centre, Mumbai (Ref no. 915-15-CR

180 [MRC]), scientific committee of the Adolfo Lutz Institute (CTC-IAL 47-J / 2017) and in

181 the Ethics Committee (CAAE: 81452517.1.0000.0059) and Ethics Committee review

182 by Universidad Peruana Cayetano Heredia (Lima, Peru) and LSHTM (London, UK).

183

\section{Sample collection}

Participating collection centres varied in their isolate collection approaches, but

the consortium collectively aimed to oversample for $M$. tuberculosis isolates with drug resistance and multi-drug resistance.

\section{Plate assay}

The CRyPTIC consortium designed two variants of the Sensititre MYCOTB

191 plate (Thermo Fisher Scientific Inc., USA) named the "UKMYC5" and "UKMYC6"

192 microtitre plates [10, 11]. These plates contain five to ten doubling dilutions of 13

193 antibiotics (rifampicin (RIF), rifabutin (RFB), isoniazid (INH), ethambutol (EMB),

194 levofloxacin (LEV), moxifloxacin (MXF), amikacin (AMI), kanamycin (KAN),

195 ethionamide (ETH), clofazimine (CFZ), linezolid (LZD), delamanid (DLM), and

196 bedaquiline (BDQ)). Delamanid and bedaquiline were provided by Otsuka

197 Pharmaceutical Co., Ltd. and Janssen Pharmaceutica respectively. The UKMYC5 
plate also contained para-aminosalicylic acid (PAS), however the MICs were not

199 reproducible and hence it was excluded from the UKMYC6 plate design and is not

200 included in any subsequent analysis [10].

A standard operating protocol for sample processing was defined by CRyPTIC as previously described $[10,11]$. Clinical samples were sub-cultured using $7 \mathrm{H} 10$ agar plates, Lowenstein-Jensen tubes or MGIT tubes. Bacterial cell suspensions $(0.5$

were diluted $100 \mathrm{X}$ in $10 \mathrm{ml}$ enriched $7 \mathrm{H} 9$ broth prior to plate inoculation. A semiautomated Sensititre Autoinoculator (Thermo Fisher, Scientific Inc., USA) was used to inoculate $100 \mu \mathrm{l}$ prepared cell suspensions $\left(1.5 \times 10^{5} \mathrm{CFU} / \mathrm{ml}\left[5 \times 10^{4} \mathrm{CFU} / \mathrm{ml}-5 \times\right.\right.$ $\left.10^{5} \mathrm{CFU} / \mathrm{ml}\right]$ ) into each well of a UKMYC5/6 microdilution plate. The plate was sealed and incubated for 14 days at $37^{\circ} \mathrm{C}$. Quality control runs were performed periodically using M. tuberculosis H37Rv ATCC 27294, which is sensitive to all drugs on the plates.

Minimum Inhibitory Concentration (MIC) measurement

As stated in reference [10], minimum inhibitory concentrations (MICs) for each

214 drug were read after incubation for 14 days by a laboratory scientist using a Thermo

215 Fisher Sensititre ${ }^{\mathrm{TM}}$ Vizion $^{\mathrm{TM}}$ digital MIC viewing system. The Vizion apparatus was

216 also used to take a high contrast photograph of the plate with a white background,

217 from which the MIC was measured again using the Automated Mycobacterial Growth

218 Detection Algorithm (AMyGDA) software [12]. The AMyGDA software detects the

219 boundaries of each well using a Hough transform for circles and measures growth as

220 the number of dark pixels within the area contained by this boundary. All images where

221 the MICs measured by Vizion and AMyGDA were different were uploaded to a citizen 
science project, BashTheBug, on the Zooniverse platform [13]. Each image was then classified by $\geq 11$ volunteers and the median classification taken. medium (either there is no plate image, or Vizion and AMyGDA disagree and there is no BashTheBug measurement), or low (all three methods disagree) quality. When considering the pattern of resistance in a sample we took the MIC from the Vizion reading, if it was annotated as having medium or low quality, to ensure adequate data coverage.

\section{Genomic data processing and variant calling}

Sequencing arrangements differed slightly between each CRyPTIC

Clockwork (v0.8.3, https://github.com/iqbal-lab-org/clockwork). Briefly, all raw sequencing files are indexed into a relational database with which Clockwork proceeds. Human, nasopharyngeal flora and human immunodeficiency virus related reads are removed and remaining reads are trimmed (adapters and low quality ends) using Trimmomatic and mapped with BWA-MEM to the M. tuberculosis H37Rv reference genome (NC000962.3) [14, 15]. Read duplicates are removed. Genetic variants are called independently using Cortex and SAMtools, two variant callers with orthogonal strengths (SAMtools a high sensitivity SNP caller, and Cortex a high specificity SNP and indel caller) $[16,17]$. The two call sets are merged to produce a

244 final call set, using the Minos adjudication tool to resolving loci where the two callers

245 disagreed, by remapping reads to an augmented genome containing each alternative 246 allele [18]. Default filters of a minimum depth of 5x and a Fraction of Supporting Reads 
of 0.9 (minos) and a Genotype confidence percentile (GCP) filter of 0.5 were applied.

248 The GCP filter is, roughly speaking, a normalised likelihood ratio test, giving a measure

249 of confidence in the called allele compared with the other alternatives, and is described

250 in [18]. This produced one VCF file per sample, each only describing positions where

251 that sample differed from the reference.

252

These filtered VCFs were then combined, to produce a single non-redundant

list of all variants seen in the cohort. All samples were then processed a second time

with Minos, remapping reads to a graphical representation of all the segregating

variation within the cohort, generating VCF files which had an entry at all variable positions (thus for all samples, most positions would be genotyped as having the reference allele).

In order to remove untrustworthy loci, a genome mask was applied to the

resulting VCF files (regions identified with self-blast matches in [19] comprising of $324,971 \mathrm{bp}$ of the reference genome. Furthermore, positions with less than $90 \%$ of total samples passing default Clockwork/Minos variant call filters (described above) with the genome mask.

\section{Phylogenetic tree construction}

A pairwise genetic distance matrix was constructed for 15,211 isolates by comparing each two variant call format (VCF) files (relative to the reference $M$. tuberculosis H37Rv genome, see [11] and the above methods section for more details) under consideration position-wise. A neighbourhood-joining tree was constructed from 
272 the distance matrix using quicktree [20]. Tree visualisation and annotation was

273 performed using the $\mathrm{R}$ library ggtree [21]. M. tuberculosis lineages were assigned

274 using Mykrobe and are represented by the coloured dots at the branch termini of the

275 tree [18]. For isolates that had 'mixed' lineage classification (i.e., 2 lineages were found

276 present in the sample by Mykrobe, $n=225,1.5 \%$ ), the first of the two lineages was

277 assigned to the isolate. ggtree was also used to construct the trees depicting

278 bedaquiline, clofazimine and delamanid resistant isolates.

\section{Data analysis and visualisation}

All data can be found in <ftp address: tbc $>$ and were analysed and visualised using either R or python3 libraries and packages. See <github: tbc> for codebase.

\section{Results}

\section{5,211 M. tuberculosis clinical isolates}

The CRyPTIC dataset comprises isolates from 27 countries collected by 14

which both genomic and phenotypic data was collected by 23 of the partner countries across the continents of Asia, Africa, South America and Europe (Fig. 1). Where the origin of an isolate was not known, the isolate was assumed to originate in the same country as the contributing laboratory (269 isolates in Germany, 17 isolates in India, 6 isolates in Peru, 885 isolates in Italy, 510 isolates in South Africa, 357 isolates in Sweden, 208 isolates in Taiwan, 1 isolate in Brazil and 4 isolates in the UK). The

294 largest number of isolates were contributed by India $(n=4,004)$, Peru $(n=2,691)$,

295 South Africa $(n=2,155)$, Vietnam $(n=1,288)$ and China $(n=1,121)$. 
four main M. tuberculosis lineages (L1-L4). Like previous studies, we see a strong association between geolocation and lineage (Pearson's chi-squared test, $p<2.2 \mathrm{e}-$ 16, Fig. S3)[22, 23]. The pie-charts in Fig. 1 and supplemental tables show the breakdown of isolate number -v- lineages (Table S2) and sub-lineages (Table S3) for each location. The phylogenetic tree in Fig. 2 further highlights the strong population structure of this collection of isolates, with isolates clustering according to lineage.

Isolates of the ancient Indo-oceanic lineage/L1 $(n=1,150)$ were mostly contributed by India ( $n=676$ isolates) and Vietnam ( $n=283$ isolates). $85 \%$ of the L1 Indian isolates belong to sub-lineages 1.1 .2 and 1.2 .2 while $66 \%$ of the Vietnamese isolates are sub-lineage 1.1.1.1. No L1 isolates were contributed by 10 of the 23 countries in this study with only 2 isolates collected in South America (one in each of

310 Brazil and Peru).

There are 5,598 L2 (East Asian) isolates, making it the second largest group in

313 the dataset. L2 was found most prevalent in Asia and Europe with the largest 314 proportion found in amongst isolates contributed by China ( $n=722,64 \%$ of isolates) 315 and India ( $n=1,573,39 \%$ of isolates). Sub-lineages 2.2 and 2.2 .7 dominate the L2 316 isolates ( $n=1,421$ and 1,249 respectively); 2.2 was found mostly amongst Peruvian 317 and South African isolates ( $n=231$ and 161 respectively) apart from those contributed 318 by the Asian countries of Vietnam $(n=271)$, China $(n=284)$ and India $(n=272)$, while $31985 \%$ of sub-lineage 2.2.7 isolates were contributed by South Africa $(n=206)$, Vietnam $320 \quad(n=164)$ and India $(n=691) .70 \%$ of sub-lineage 2.2 .1 originated from South Africa 321 (10\% of isolates found here) and has recently been associated with more favourable 
322 transmission rates [24]. Lastly, $86 \%$ and $72 \%$ of isolates contributed by Kyrgyzstan

323 and Turkmenistan respectively belong to $L 2$ with sub-lineage 2.2 .10 dominating (16/24

324 and 75/86 isolates for both countries respectively). 2.2.10 has been previously

325 described as restricted to Central Asia and this is reflected in the compendium [25].

The majority $(1184 / 1850,65 \%)$ of L3 (East African/Indian) isolates were

328 contributed by India, followed by $19.6 \%(363 / 1850)$ isolates from Pakistan. L3 is typically under-sampled and under-studied in current databases and biobanks in

330 comparison to L2 and L4; the L3 isolates in this study are the largest collected to date 331 in a single study [26].

334 Isolates donated from Peru dominate; $87 \%$ of all L4 isolates are Peruvian with 4.1.2.1

335 and 4.3 .3 being the most prevalent sub-lineages ( $24 \%$ and $22 \%$ respectively). There

336 are 34 different L4 sub-lineages in the dataset, making L4 the most diverse in 337 comparison to the other lineage groupings.

340 identified. Animal-restricted pathogenic mycobacterial isolates are also rare in the

341 compendium; only 16 cases were identified ( $n=15 \mathrm{M}$. bovis and $n=1 \mathrm{M}$. caprae). 
Figure 1: Geographical distribution of CRyPTIC M. tuberculosis clinical isolates. The total number of isolates contributed by each country is depicted, with pie charts representing the proportion of $M$. tuberculosis lineages. Where the origin of an isolate was not known, the collection site identity was assigned (269 isolates in Germany, 17 isolates in India, 6 isolates in Peru, 885 isolates in Italy, 510 isolates in South Africa, 357 isolates in Sweden, 208 isolates in Taiwan, 1 isolate in Brazil and 4 isolates in the UK). 
Figure 2: Phylogenetic tree of CRyPTIC M. tuberculosis clinical isolates. A phylogenetic cladogram of 15,211 M. tuberculosis clinical isolates. A neighbour-joining tree was constructed from a pairwise distance matrix using quicktree [20]. Coloured dots at the branch termini represent the lineage assigned to each isolate. "Animal/Other" includes 16 isolates that were assigned the following lineages: M. caprae, M. bovis, unknown along with 17 quality control isolates representing specific sub-lineages [27].

\section{Quality assurance of the drug minimum inhibitory concentrations}

Regular quality assurance checks detected problems with plate inoculation and reading in two laboratories during collection of the 15,211 isolates. In addition, some plates were not able to be read, usually because of inadequate growth in the positive control wells or extensive contamination. These anomalies were investigated, and samples were removed from the dataset where appropriate, resulting in 2,922 isolates being held out of the final dataset. Further, due to skip wells and other phenomena that prevent an MIC being measured, $11.9 \%$ of isolates did not return a phenotype for all 13 drugs on the plate. The compendium therefore has a total of $157,401 \mathrm{MIC}$ measurements for 12,289 isolates (Table 1). 


\begin{tabular}{c|cccc}
\multicolumn{1}{c}{} & MIC & HIGH QUALITY & MEDIUM QUALITY & LOW QUALITY \\
\hline INH & 12070 & 9519 & 1351 & 1200 \\
RIF & 12099 & 8955 & 1356 & 1788 \\
EMB & 12158 & 7506 & 1355 & 3297 \\
LEV & 12163 & 7774 & 1354 & 3035 \\
MXF & 12194 & 6785 & 1353 & 4056 \\
AMI & 12072 & 8973 & 1350 & 1749 \\
KAN & 12130 & 9333 & 1355 & 1442 \\
BDQ & 12068 & 8536 & 1355 & 2177 \\
CFZ & 12049 & 7763 & 1352 & 2934 \\
DLM & 11927 & 8095 & 1349 & 2483 \\
LZD & 12189 & 7141 & 1355 & 3693 \\
ETH & 12132 & 8821 & 1355 & 1956 \\
RFB & 12150 & 10042 & 1352 & 756 \\
TOTAL & $\mathbf{1 5 7 4 0 1}$ & $\mathbf{1 0 9 2 4 3}$ & $\mathbf{1 7 5 9 2}$ & $\mathbf{3 0 5 6 6}$
\end{tabular}

Table 1: Quality metrics for phenotype data Stated for each drug is the total number of MIC readings ("PHENOTYPED") stratified into "high" quality (at least two MIC measurement methods agree), "medium" quality (either Vizion and AMyGDA disagree, or there is no plate picture) or "low" quality (all three MIC measurements methods disagree) phenotype classifications as described in Methods. Drug acronyms: INH = isoniazid, RIF = rifampicin, EMB = ethambutol, $\mathrm{LEV}=$ levofloxacin, $\mathrm{MXF}=$ moxifloxacin, $\mathrm{AMI}=$ amikacin, $\mathrm{KAN}=$ kanamycin, $\mathrm{BDQ}=$ bedaquiline, $\mathrm{CFZ}=$ clofazimine, $\mathrm{DLM}=$ delamanid, $\mathrm{LZD}=$ linezolid, $\mathrm{ETH}=$ ethionamide, $\mathrm{RFB}=$ rifabutin.

Resistance classification and distribution applying an epidemiological cut-off value (ECOFF); samples with MICs at or below the ECOFF are, by definition, wild-type and hence assumed to be susceptible to the drug in question [11] (Fig. S1). Samples with MICs above the ECOFF are therefore assumed to be resistant. within the CRyPTIC compendium (Fig. 3a). The drugs with the highest percentage of

388 resistance are the first line drugs isoniazid and rifampicin $(49.0 \%$ and $38.7 \%$ respectively). Of the second line drugs, levofloxacin had the highest proportion of 
resistant isolates in the dataset (17.6\%) and amikacin the lowest (7.3\%). Reassuringly,

a low proportion of isolates were resistant to the NRDs, bedaquiline $(0.9 \%)$, clofazimine $(4.4 \%)$, delamanid $(1.6 \%)$ and linezolid $(1.3 \%)$.

Of the 12,289 isolates which returned a binary phenotype for at least one drug, $6,814(55.4 \%)$ were 'resistant' to at least one drug (Fig. 3b). For the purpose of describing the broader resistance categories present in the dataset, we assumed that all MICs that could not be read had susceptible phenotypes in this section. Consequently, the calculated prevalence of MDR, XDR etc in the dataset (Fig. 3) are likely under-estimates.

At least $68.8 \%(n=4,685)$ of drug resistant isolates were either MDR or RR (Fig. 3b) and of these 38.8\% $(n=1,819)$ were pre-XDR (MDR/RR + fluoroquinolone resistance) and $3.0 \%$ were XDR $(n=142)$. Two of the XDR isolates returned a resistant phenotype to all 13 of the drugs assayed (Table S1) and therefore could be reasonably described as totally drug resistant (TDR). One such isolate belonged to L4 and was contributed by South Africa, and the other belonged to $L 2$ with an unknown country of origin.

Of the 2,129 resistant isolates that did not fit into either of the MDR or RR categories, more were categorised as isoniazid resistant $(73.1 \%, n=1,556)$ than were

411 categorised as susceptible to both isoniazid and rifampicin and resistant to at least 412 one other drug $(26.9 \%, n=573)$. 
Whilst many partner laboratories oversampled for resistance, others used a

415 less biased collection protocol (Fig. 3c). Therefore, the proportions of resistant

416 phenotypes are not necessarily representative of the overall distribution of resistance

417 in the country and are not comparable between countries. Isolates with unknown

418 country of origin were excluded from this analysis. In all countries that contributed

419 more than 100 resistant isolates, each of the five phenotypic resistance categories in

420 Fig. 3b were identified, except for Peru, Vietnam and Nepal which did not contribute

421 any XDR isolates. Vietnam and Brazil sampled a particularly high proportion of non-

422 MDR/RR resistant phenotypes; $73.9 \%$ and $55.1 \%$ of resistant isolates contributed by

423 these countries, respectively, were neither MDR nor RR.

For Nepal and India, an especially high proportion of the MDR/RR isolates

contributed were fluoroquinolone resistant $(92.9 \%(n=132 / 142$ isolates $)$ and $69.8 \%$ ( $n=538 / 770$ isolates) respectively), as has been previously observed for this geographical region [28, 29].

All five categories of resistance were represented in the four major $M$. tuberculosis lineages. We note that these relative proportions will have been

432 influenced by the different sampling approaches at each partner laboratory since

433 lineage distributions are typically geographically distinct (Fig. 1). Bearing this in mind, 434 we observe that in the compendium, L3 isolates contained the most MDR/RR isolates 435 as a proportion of resistant isolates (77.6\%), followed by L2 (74.3\%), L4 (63.4\%), and 436 L1 (50.0\%). L2 is known to be associated with MDR/RR and XDR and although it did 437 not contain the highest proportion of MDR/RR isolates, it did have the most pre-XDR 438 isolates as a proportion of MDR/RR isolates $(54.2 \%)[30,31]$. L1 and L2 contained the 
bioRxiv preprint doi: https://doi. org/10.1101/2021.09.14.460274. this version posted September 16, 2021. The copyright holder for this preprint (which was not certified by peer review) is the author/funder, who has granted bioRxiv a license to display the preprint in perpetuity. It is made available under aCC-BY 4.0 International license.

a

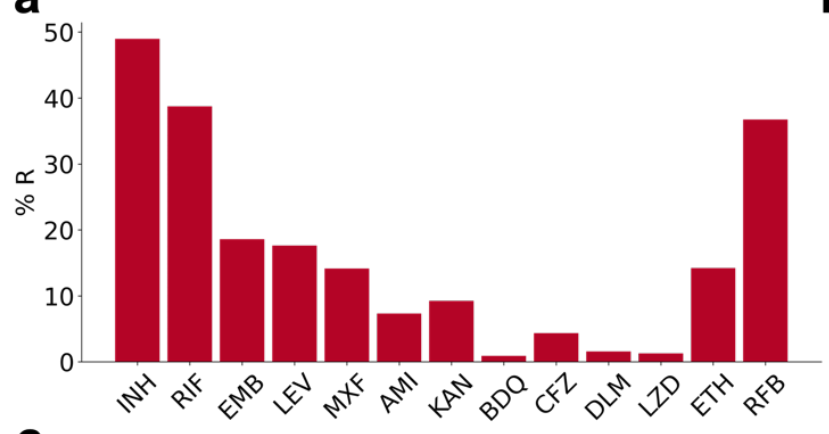

C

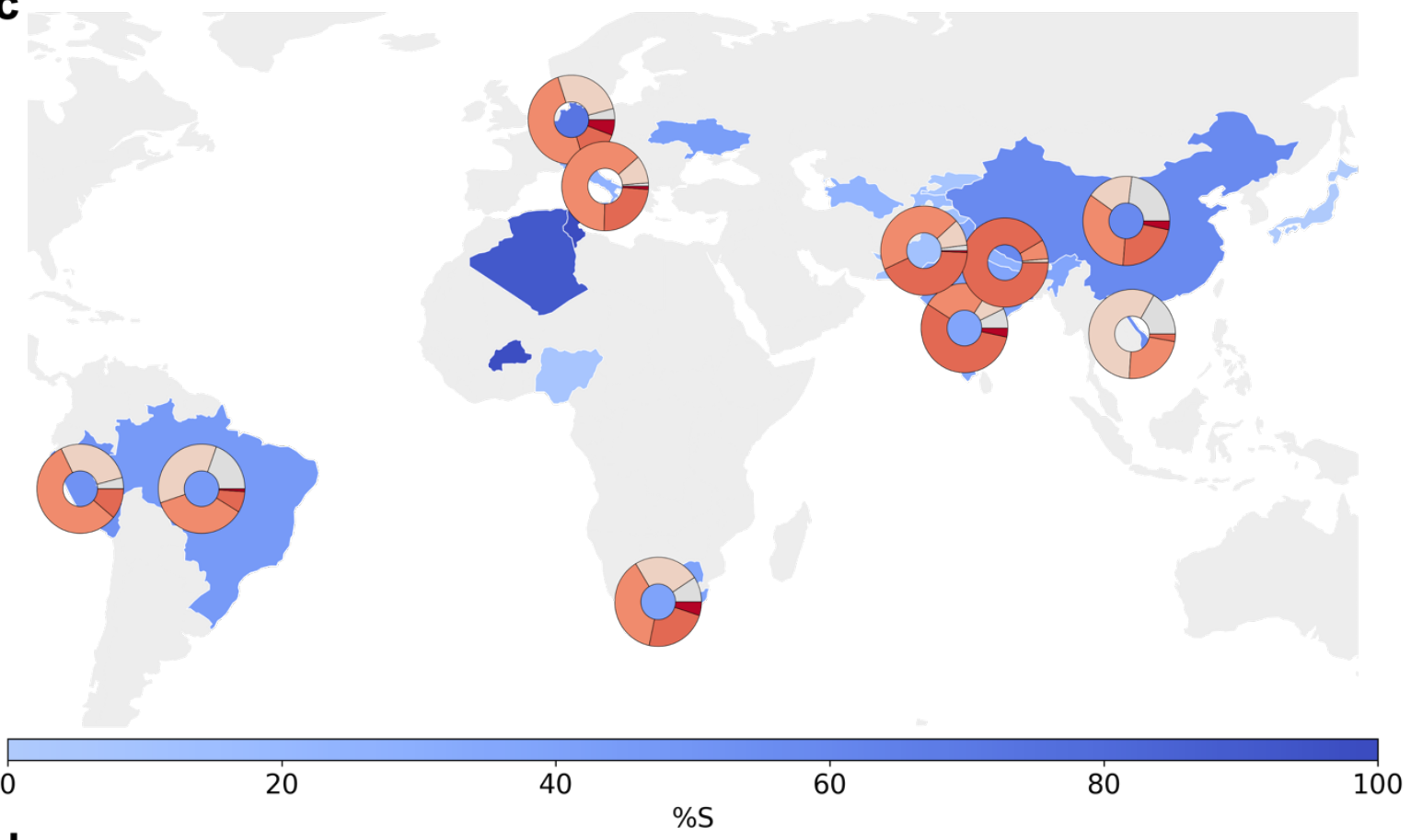

b

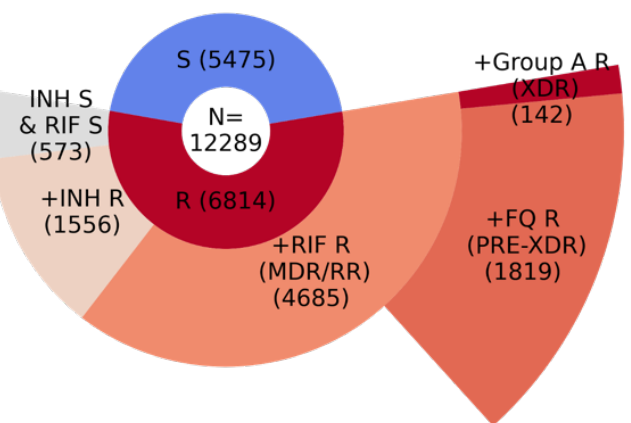

d

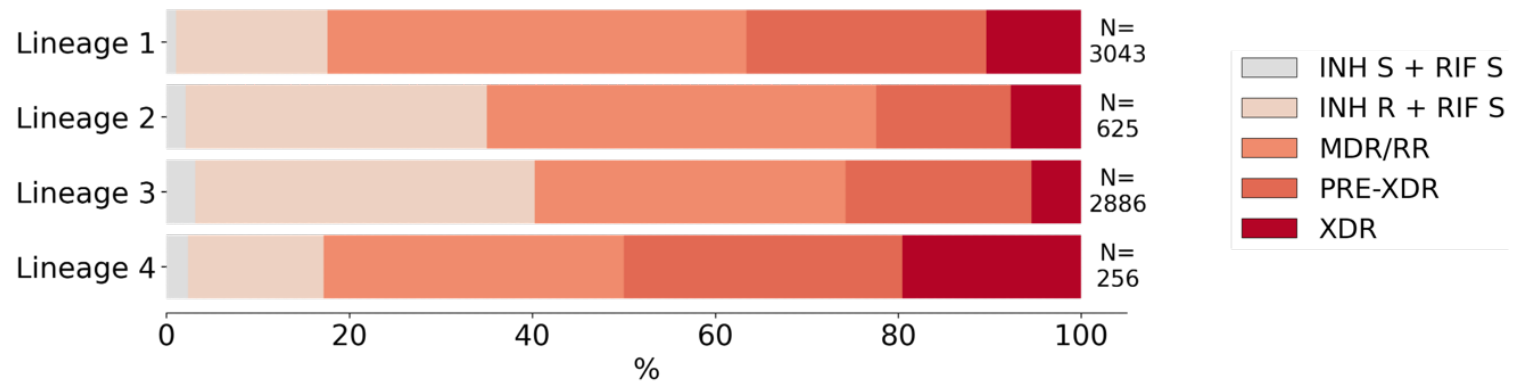

Figure 3: Drug phenotype data for the CRyPTIC compendium a) Prevalence of resistance to each of 13 drugs in the CRyPTIC dataset. The total number of isolates with a binary phenotype (of any quality) for the corresponding drug is presented in Table 1. b) Phenotypes of 12, 289 CRyPTIC isolates with a binary phenotype for at least one drug. c) Geographical distribution of phenotypes of 12, 289 CRyPTIC isolates. Intensity of blue shows the percentage of isolates contributed that were categorised as susceptible to all 13 drugs. Donut plots show the 
proportions of resistant phenotypes identified in (b) for countries contributing $>=100$ isolates with drug resistance. d) Proportions of resistance phenotypes in the 4 major $M$. tuberculosis lineages. $\mathrm{N}$ is the number of isolates of the lineage called resistant to at least one of the 13 drugs. Drug acronyms: $\mathrm{INH}=$ isoniazid, RIF = rifampicin, EMB = ethambutol, LEV = levofloxacin, $\mathrm{MXF}=$ moxifloxacin, $\mathrm{AMI}=$ amikacin, $\mathrm{KAN}=$ kanamycin, $\mathrm{BDQ}=$ bedaquiline, $\mathrm{CFZ}=$ clofazimine, $\mathrm{DLM}=$ delamanid, LZD = linezolid, ETH = ethionamide, RFB = rifabutin.

\section{Co-occurrence of drug resistance amongst the CRyPTIC isolates}

Isolates with all possible two-drug resistant combinations were present in this dataset (Fig. 4a). Resistance to any of the remaining 11 drugs was associated with resistance to both isoniazid and rifampicin. Isoniazid resistance was the most strongly associated bar the following two exceptions, both of which arise because drugs from the same class are being compared: a greater proportion of isolates resistant to rifabutin were also resistant to rifampicin $(96.8 \%$ had rifabutin - rifampicin coresistance versus $93.3 \%$ rifabutin - isoniazid co-resistance) and a greater proportion of isolates resistant to moxifloxacin were associated with levofloxacin resistance

466 (97.6\% had moxifloxacin - levofloxacin co-resistance versus $95.0 \%$ moxifloxacin isoniazid co-resistance). Of the second line drugs, levofloxacin and moxifloxacin were more commonly seen as a second resistant phenotype than the injectable drugs kanamycin and amikacin.

Despite this, resistance to both drugs in the aminoglycoside class was common

472 the dataset; $90.4 \%$ of amikacin resistant isolates were also resistant to kanamycin although significantly fewer kanamycin resistant isolates were resistant to amikacin

474 (72.0\%, $p<0.00001)$. In a similar fashion, a smaller proportion of rifampicin resistant 475 isolates were resistant to rifabutin than rifabutin resistant isolates that were resistant 476 to rifampicin $(91.3 \%, 96.8 \%, p<0.00001)$ while a smaller proportion of levofloxacin 
resistant isolates were resistant to moxifloxacin than moxifloxacin resistant isolates

478 that were resistant to levofloxacin $(78.5 \%, 97.6 \%, p<0.00001)$. One possible explanation is that the cut off, in this case the ECOFFs, have been inconsistently

480 defined for the different compounds, however here all ECOFFs have been determined

481 in the same way using a much larger dataset than is typically the case [11]. Differences

482 in drugs of the same class are also well documented by in vitro studies [32-34]. a second resistance phenotype and the smallest proportional resistance combinations involved the NRDs (e.g. 1.5\% of isoniazid resistant isolates were bedaquiline resistant and $1.7 \%$ were delamanid resistant). Within the NRDs however, co-occurrence of resistance was proportionally higher; bedaquiline resistance was most seen with clofazimine resistance $(52.4 \%)$, clofazimine resistance was most seen with bedaquiline resistance $(10.6 \%)$, delamanid resistance was most seen with clofazimine resistance (26.3\%) and linezolid resistance was most seen with clofazimine resistance $(34.2 \%)$

Additional antibiotic resistance in isolates with MDR or non-MDR phenotypic

\section{backgrounds}

We next examined in more detail the correlation structure of phenotypes by conditioning on different phenotypic "backgrounds". (Fig. 4b-f). We found that a greater proportion of isolates that were susceptible to isoniazid and rifampicin were

500 resistant to second-line drugs than the first line drug ethambutol (15.9\%) (Fig. 4b). The 501 proportion of isolates resistant to clofazimine or levofloxacin was particularly high 
502 (32.9\% and $24.1 \%$, respectively), and more isolates were resistant to these two drugs

503 than ethambutol in an isoniazid resistant and rifampicin susceptible background but

504 not in MDR/RR isolates (Fig. 4c-f).

505

MDR/RR isolates (asides from rifabutin) were most commonly resistant to the

507 first line drug ethambutol (46.3\%), closely followed by levofloxacin $(41.4 \%)$, then moxifloxacin (34.1\%). A greater proportion of isolates resistant to levofloxacin than moxifloxacin was also seen in all other backgrounds and as expected, the proportion

510 of fluoroquinolone resistance was higher in MDR/RR isolates than non-MDR isolates

511 [35]. For the aminoglycosides, a greater percentage of $M D R / R R$ isolates were 512 kanamycin resistant $(21.8 \%)$ than amikacin resistant $(18.1 \%)$, a trend seen in all other 513 backgrounds.

For isolates with an XDR phenotype, a higher proportion were also resistant to

516 linezolid than bedaquiline (66.7\% compared to $44.6 \%)$ and $11.3 \%$ of XDR isolates

517 were resistant to both bedaquiline and linezolid (Fig. 4f). XDR isolates were also 518 resistant to the other NRDs, clofazimine (41.3\%) and delamanid (18.8\%). For isolates

519 with an MDR/RR or pre-XDR background, the most common NRD resistance seen 520 was clofazimine $(6.0 \%$ and $9.3 \%$, respectively) followed by linezolid $(2.3 \%$ and $4.7 \%$

521 respectively), bedaquiline (1.8\% and $3.2 \%$, respectively), and delamanid (1.7\% and $5222.3 \%$, respectively). In non-MDR/RR backgrounds: clofazimine resistance remained 523 the most common followed by delamanid resistance, linezolid resistance and 524 bedaquiline resistance (Fig. 4b-c). 

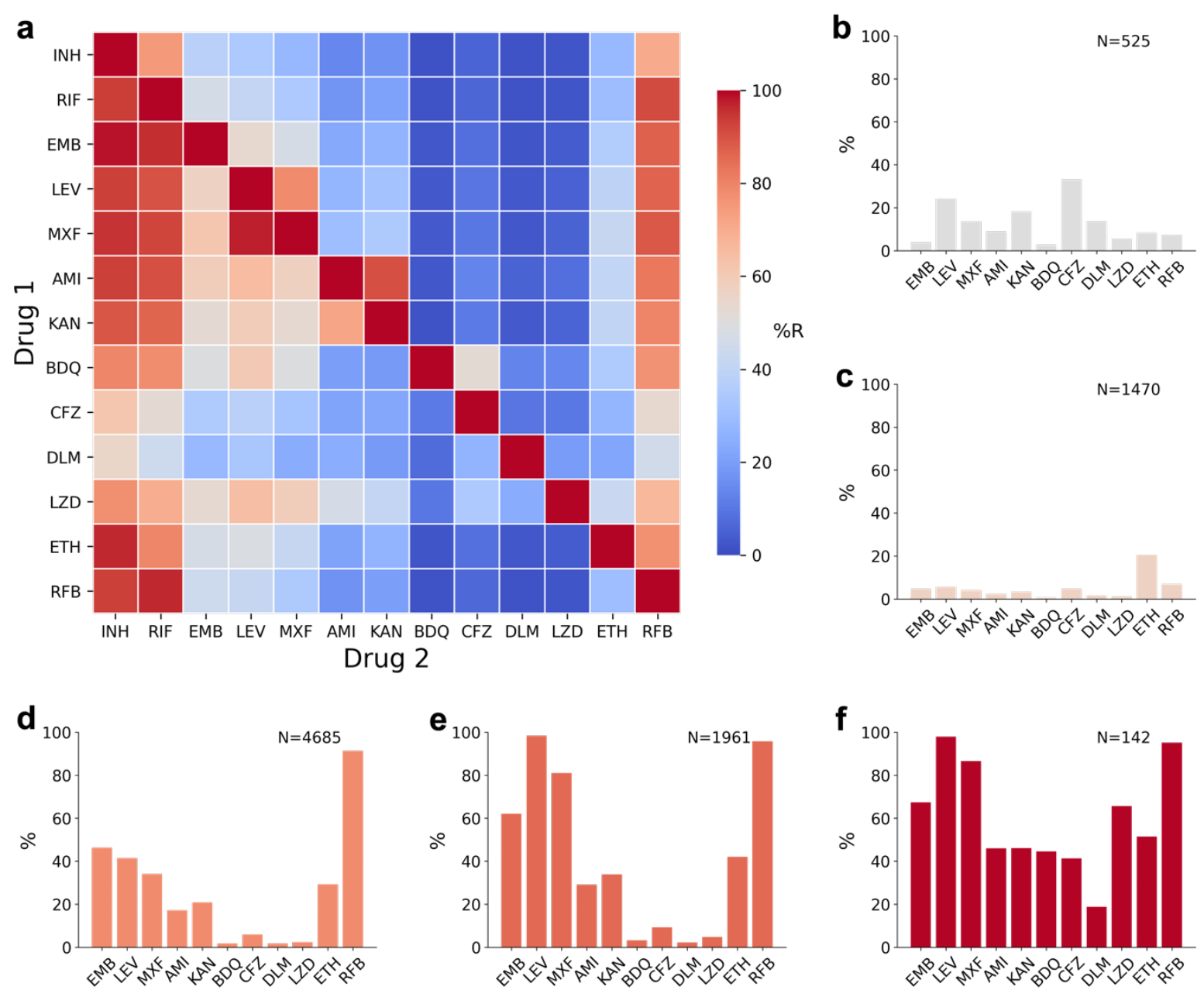

Figure 4: Co-occurrence of antibiotic resistance in CRyPTIC M. tuberculosis isolates a) The heatmap shows the probability of an isolate being resistant to Drug 2 if it is resistant to Drug 1, percentages are given in Table S4. (b-g) Percentage of isolates that are resistant to another of the 13 drugs in a background of (b) isoniazid susceptible + rifampicin susceptible, (c) isoniazid resistant + rifampicin susceptible, (d) MDR/RR (e) Pre-XDR, (f) XDR. Only samples with definite phenotypes for RIF in MDR backgrounds and RIF and INH in non-MDR backgrounds and the additional drug are included. Drug acronyms: INH = isoniazid, RIF = rifampicin, $\mathrm{EMB}=$ ethambutol, $\mathrm{LEV}=$ levofloxacin, $\mathrm{MXF}=$ moxifloxacin, $\mathrm{AMI}=$ amikacin, $\mathrm{KAN}=$ kanamycin, $\mathrm{BDQ}=$ bedaquiline, $\mathrm{CFZ}=$ clofazimine, $\mathrm{DLM}=$ delamanid, $\mathrm{LZD}=$ linezolid, $\mathrm{ETH}=$ ethionamide, RFB = rifabutin.

Genetic determinants of resistance

In order to establish a baseline measure of how well resistance and project, we constructed a hybrid catalogue of genetic variants associated with resistance to the first-line compounds isoniazid, rifampicin \& ethambutol and the 
second-line compounds levofloxacin, moxifloxacin, amikacin, kanamycin, ethionamide based on existing catalogues $[7,36]$. (We note in passing that we specifically did not

547 use the recent $\mathrm{WHO}$ catalogue in order to avoid circularity and over-training, as that 548 catalogue was developed (via prior literature, expert rules and a heuristic algorithm)

549 based partially on these isolates [37]. We applied our hybrid catalogue to predict the susceptibility and resistance of all isolates in this compendium to these compounds, and the resulting genetic-based predictions were then compared with the binary phenotypes derived from the MICs (Table 2). Since these data were not collected prospectively or randomly, but indeed are enriched for resistance, the calculated error rates are not representative of how well such a method would perform in routine clinical use. We also did not apply the approach used in [7] of refusing to make a prediction if a novel mutation was detected in a known resistance gene, as we simply wanted to measure how well a pre-CRyPTIC catalogue could predict resistance in the compendium. The results were broadly in line with prior measurements on a smaller (independent) set [18]. The hybrid catalogue does not make predictions for rifabutin,

560 linezolid, bedaquiline, delamanid or clofazimine; indeed, this is one of the main aims of the consortium and new catalogues published by CRyPTIC and the WHO will begin to address this shortcoming (Fig. 5) [19].

Table 3 shows the top mutations found amongst isolates phenotypically

566 resistant to first- and second- line drugs. As expected, rpoB S450L is the most 567 prevalent mutation associated with rifampicin resistance, with $62.2 \%$ of all 568 phenotypically rifampicin-resistant isolates carrying it, while $70 \%$ of isoniazid resistant 569 isolates have katG S315T mutations. Mutations in gyrA dominate amongst 570 fluoroquinolone resistant isolates; D94G and A90V are the two most frequently 
occurring mutations for levofloxacin and moxifloxacin, with just under $77 \%$ and just respectively. Mutations in $r p o C$ are amongst those most prevalent for rifabutin

574 resistant isolates; these mutations are typically described as compensatory, while 575 G594E was subsequently defined as a lineage-specific marker for L4 LAM and 576 Haarlem lineages [38].

577

\begin{tabular}{c|cccccccc}
\multicolumn{1}{c}{} & TP & FP & TN & FN & VME & ME & PPV & NPV \\
\hline INH & 5493 & 142 & 5622 & 224 & 0.039 & 0.025 & 0.961 & 0.975 \\
RIF & 4535 & 435 & 6669 & 107 & 0.023 & 0.061 & 0.977 & 0.939 \\
EMB & 1919 & 513 & 6702 & 111 & 0.055 & 0.071 & 0.945 & 0.929 \\
LEV & 1689 & 255 & 8104 & 184 & 0.098 & 0.031 & 0.902 & 0.969 \\
MXF & 1358 & 504 & 9022 & 160 & 0.105 & 0.053 & 0.895 & 0.947 \\
AMI & 632 & 84 & 10117 & 163 & 0.205 & 0.008 & 0.795 & 0.992 \\
KAN & 735 & 124 & 9043 & 197 & 0.211 & 0.014 & 0.789 & 0.986 \\
ETH & 971 & 114 & 9183 & 511 & 0.345 & 0.012 & 0.655 & 0.988
\end{tabular}

\section{Table 2: Predicting phenotypic resistance using genetics}

580 TP: the number of phenotypically resistant samples are that correctly identified as resistant ("true positives"); FP, the number of phenotypically susceptible samples that are falsely identified as resistant ("false positives"); TN, the number of phenotypically susceptible samples that are correctly identified as susceptible ("true negatives"); FN, the number of phenotypically resistant samples that are incorrectly identified as susceptible ("false negative"); VME, very major error rate (false-negative rate), $0-1$; ME, major error rate (false-positive rate), 0-1; PPV, positive predictive value, $0-1$; NPV, negative predictive value, $0-1$. 


\begin{tabular}{|c|c|c|c|c|}
\hline & GENE & VARIANT & $n$ & $\%$ \\
\hline \multirow[t]{5}{*}{ RIF } & $r p o B$ & S450L & 2914 & 62.2 \\
\hline & rpoB & D435V & 506 & 10.8 \\
\hline & rроB & H445D & 202 & 4.3 \\
\hline & rpoB & H445Y & 147 & 3.1 \\
\hline & rpoB & D435Y & 112 & 2.4 \\
\hline \multirow[t]{5}{*}{ INH } & katG & S315T & 3668 & 70. \\
\hline & $f a b G 1$ & $c-15 t$ & 829 & 15.8 \\
\hline & $f a b G 1$ & $g-17 t$ & 176 & 3.4 \\
\hline & $f a b G 1$ & $t-8 c$ & 154 & 2.9 \\
\hline & $\operatorname{inh} A$ & I194T & 56 & 1.1 \\
\hline \multirow[t]{5}{*}{ EMB } & $e m b B$ & M306V & 1131 & 50 \\
\hline & $e m b B$ & M306I & 1001 & 44.3 \\
\hline & $e m b B$ & Q497R & 449 & 19.9 \\
\hline & $e m b B$ & G406A & 164 & 7.2 \\
\hline & $e m b B$ & G406D & 105 & 4.6 \\
\hline \multirow[t]{3}{*}{ KAN } & rrs & a1401g & 660 & 58.9 \\
\hline & eis & $c-14 t$ & 70 & 6.2 \\
\hline & eis & g-10a & 53 & 4.7 \\
\hline \multirow[t]{2}{*}{ AMI } & rrs & a1401g & 660 & 74.7 \\
\hline & $r r s$ & g1484t & 7 & 0.8 \\
\hline \multirow[t]{5}{*}{ LEV } & gyrA & D94G & 783 & 36.5 \\
\hline & gyrA & A90V & 487 & 22.7 \\
\hline & gyrA & D94N & 157 & 7.3 \\
\hline & gyrA & D94A & 133 & 6.2 \\
\hline & gyrA & S91P & 92 & 4.3 \\
\hline \multirow[t]{5}{*}{ MXF } & gyrA & A90V & 487 & 28.2 \\
\hline & gyrA & D94G & 783 & 45.4 \\
\hline & gyrA & D94N & 157 & 9.1 \\
\hline & gyrA & D94A & 133 & 7.7 \\
\hline & gyrA & D94Y & 70 & 4.1 \\
\hline \multirow[t]{2}{*}{ ETH } & $f a b G 1$ & $c-15 t$ & 829 & 48 \\
\hline & $f a b G 1$ & L203L & 124 & 7.2 \\
\hline
\end{tabular}

598 Table 3: The top mutations associated with phenotypic drug resistance 599 A genetic resistance catalogue was used for genotypic prediction of resistance for each drug in 600 column 1 [7, 36]. "VARIANT": non-synonymous amino acid mutations are denoted by upper case 601 letters while nucleotide substitutions for non-coding sequences are denoted by lower case letters. 602 Negative numbers denote substitutions in promoter regions; "GENE": genic region of interest in 603 which "Variant" can be found; " $n$ ": number of phenotypically resistant isolates with "VARIANT"; "\%": 604 percentage of total phenotypically resistant isolates with "VARIANT". Drug acronyms: INH = 605 isoniazid, RIF = rifampicin, EMB = ethambutol, $\mathrm{AMI}=$ amikacin, $\mathrm{KAN}=$ kanamycin, $\mathrm{LEV}=$ 606 levofloxacin, MXF = moxifloxacin, $\mathrm{ETH}=$ ethionamide. 


\section{Resistance to new and re-purposed drugs}

611 As previously stated, relatively few isolates are resistant to the NRDs,

612 bedaquiline $(n=109)$, clofazimine $(n=525)$, delamanid $(n=186)$ and linezolid $(n=$

613 156). South Africa contributed the greatest number of isolates resistant to bedaquiline,

614 clofazimine and linezolid (Fig. 5a), while China and India contributed the most isolates

615 resistant to delamanid. Since the collection protocol differed between laboratories it is

616 not possible to infer any differences in the relative prevalence of resistance to the

617 NRDs in these countries. The results of a survey of all non-synonymous mutations in

618 genes known or suspected to be involved in resistance to these four drugs (e.g.

$619 r v 0678, m m p L 5, p e p Q, d d n, r p / C, r r l$ etc.) are depicted in Fig. 5b-e [39-43]. Mutations

620 known to be associated with sensitivity were ignored, along with mutations that

621 occurred at a frequency of $\geq 5 \%$ amongst all isolates. In contrast to first- and second-

622 line drugs, there are no mutations within a single gene/small group of genes that can

623 fully explain resistance to any NRD. Note that the role of most of these mutations in

624 resistance remains undetermined. Work is ongoing within the consortium to unravel

625 the association of these (and novel) mutations with resistance to the NRDs along with

626 the relevant mechanisms of resistance. 
b
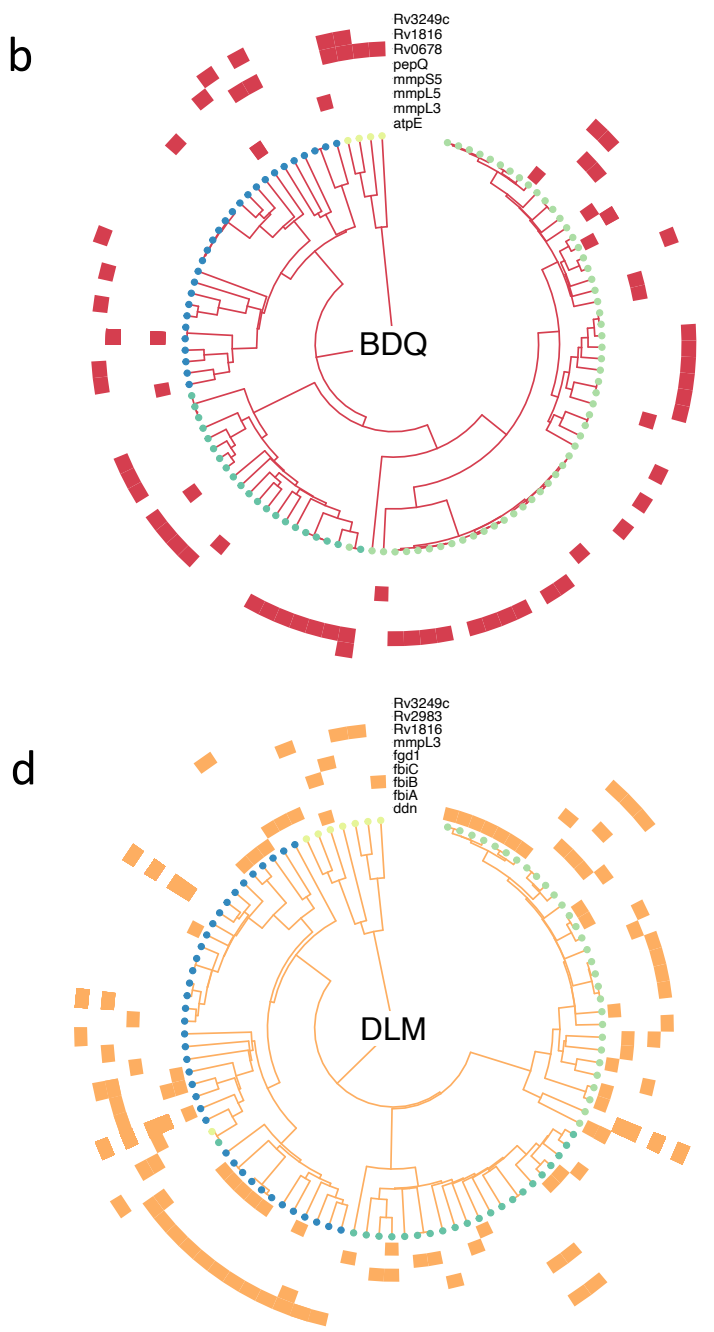

C

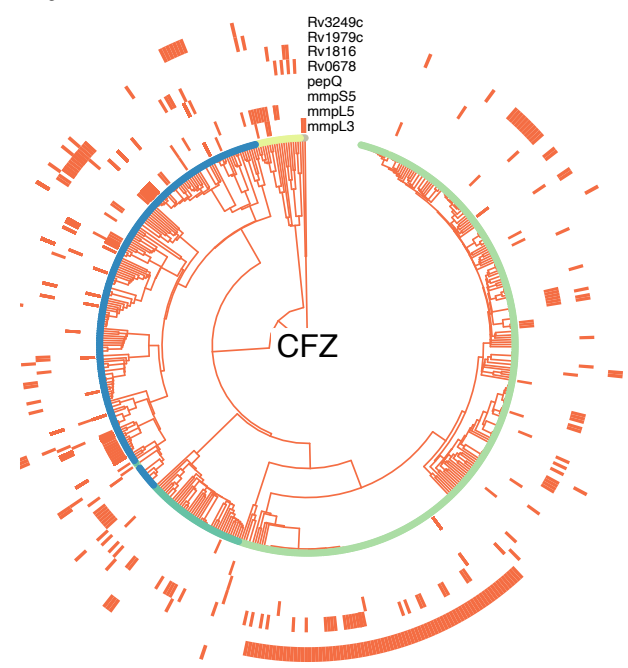

e

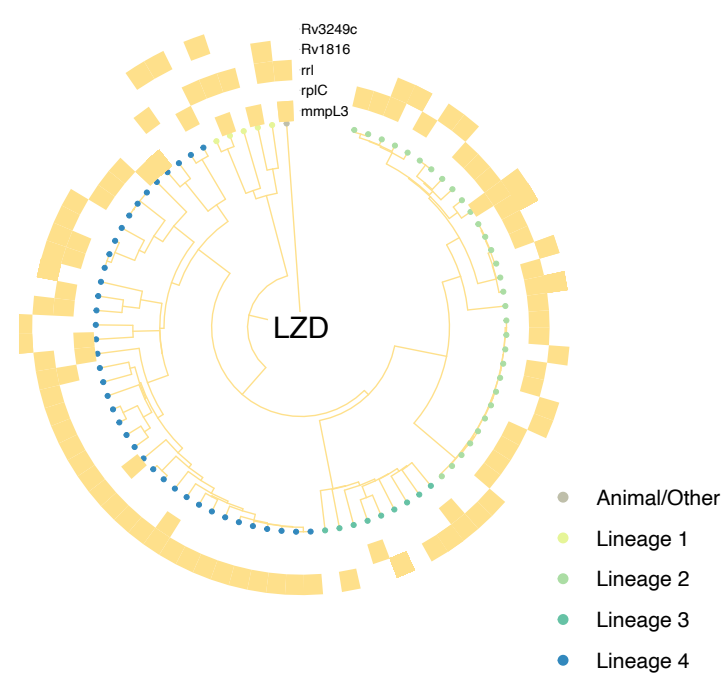

629 Figure 5: Resistance to bedaquiline, clofazimine, delamanid and linezolid 630 amongst $M$. tuberculosis CRyPTIC isolates a) The prevalence of resistance to 631 bedaquiline (BDQ), clofazimine (CFZ), delamanid (DLM) and linezolid (LZD) per country or origin 632 or collection site. Phylotrees are shown for isolates phenotypically resistant to b) BDQ, c) CFZ, d) 633 DLM and e) LZD. Tip point colours denote lineage. Each outer track represents a gene thought to 
634 be associated with resistance and coloured blocks denote the presence of a non-synonymous

635 mutation in the relevant gene for a given isolate. Mutations in these genes that are either associated with sensitivity or present in $>5 \%$ of the collection of isolates as a whole were ignored.

\section{Case study on rifampicin mono-resistance}

is increasing $[1,44]$. The WHO does not recommend isoniazid for RMR treatment, despite it being effective; this is likely due to the reliance on the Xpert® MTB/RIF assay which cannot distinguish between RMR and MDR. Use of isoniazid could improve treatment outcomes for RMR patients which are currently similar to that of MDR TB, including a higher risk of death compared to drug susceptible infections [45, 46]. Due to its low natural prevalence, RMR has been poorly studied to date but increasingly large clinical TB datasets, such as the one presented here, make its study now feasible. database that also had a phenotype for isoniazid, 302 (6.5\%) were RMR. These isolates were contributed by South Africa, Peru, India, Italy, China, Pakistan, Brazil,

655 Vietnam, Germany, Nigeria, Turkmenistan and Tajikistan (Fig. 6a). South Africa and 656 Nigeria contributed a significantly higher number of RMR isolates, as a proportion of rifampicin resistant isolates, than that of the total dataset at $17.5 \%(p<0.00001)$ and $27.3 \%(p=0.00534)$ respectively. We note that countries may have oversampled for solo resistance, but the higher contribution of RMR isolates from South Africa is 
RMR has evolved in all four major lineages, with L4 having a significantly higher

663 RMR prevalence than that of the total rifampicin resistant dataset at $9.4 \%(p=$ $6640.00031)$ and L2 significantly lower RMR prevalence at $4.2 \%(p=0.00026)$. Although 665 these numbers will have been somewhat influenced by differences in sample 666 collection bias at different geographical sites, we would expect L2 to have a lower 667 proportion of RMR isolates due to its association with MDR phenotypes [31, 47] (Fig. 668 6b).

Rifampicin mono-resistance is incorrectly predicted by current diagnostics

A widely used, WHO-endorsed diagnostic tool, the Xpert@ MTB/RIF assay, uses a proxy whereby any SNP detected in the "rifampicin-resistance determining region" (RRDR) of $r p o B$ results in a prediction of MDR. However, the suitability of the

674 proxy is dependent upon prevalence of RMR in the population [44]. We tested the 675 reliability of this on the 4,655 rifampicin resistant isolates in our dataset that had a phenotype for isoniazid (Fig. 6c). the MDR isolates had no RRDR mutation and therefore $4.0 \%$ of isolates in this study 680 would be predicted as false negative MDR by the Xpert@ MTB/RIF assay. 276 of the 681 RMR isolates had a mutation in the RRDR of $r p o B$ and so the Xpert@ MTB/RIF assay 682 proxy would incorrectly predict $5.9 \%$ of the rifampicin resistant isolates as false 683 positive MDR cases. However, overall, the Xpert® MTB/RIF assay proxy correctly 684 predicts $89.5 \%$ of the rifampicin resistant isolates as MDR and $0.6 \%$ of the isolates as 685 non-MDR in this dataset, which suggests it is a reasonably successful diagnostic tool 
with $>90 \%$ accuracy for MDR classification of rifampicin resistant isolates. As most sites oversampled for resistance, our dataset likely contains a higher prevalence of RMR than the global average and hence the Xpert $\circledast$ MTB/RIF assay is likely to perform better on more representative data. However, the analysis shows how the increasing global levels of RMR TB cases could increase the number of false positive MDR diagnoses by the Xpert ${ }^{\circledR}$ MTB/RIF assay, denying isoniazid treatment to a greater number of patients who would then be moved on to less effective drugs.

\section{There are genetic differences between rifampicin mono-resistant and}

\section{multidrug resistant isolates}

We have analysed our matched phenotypic and genotypic data to examine

697 whether there were any differences in the genetic determinants of rifampicin resistance between RMR and MDR isolates. The proportion of RMR isolates with no rpoB mutation (5.3\%, Fig. $6 \mathrm{c})$ was significantly higher than that of MDR isolates $(1.8 \%$, $p<0.00001)$. This suggests that non-target-mediated resistance mechanisms, such as upregulation of rifampicin specific efflux pumps, could be more influential in providing protection against rifampicin in RMR isolates than in MDR isolates.

The majority of RMR and MDR isolates contained one or more SNPs in rpoB, with the majority having at least one mutation in the RRDR. To date, several nonsynonymous RRDR mutations have been found in RMR M. tuberculosis isolates,

707 including the resistance conferring mutations S450L, H445D and D435Y, which are also seen in MDR isolates $[48,49]$. For both RMR and MDR isolates in this dataset, the most common rpoB RRDR mutation seen was S450L $(63.6 \%$ and $41.1 \%$ of

710 isolates respectively, Fig. 6d). Five mutations were present in RMR isolates that were 
711 not seen in MDR isolates: S428G, S441A, S441V, S450M and S450Q, however these

712 were seen at low prevalence (less than $2 \%$ ) of RMR isolates. We found more RMR

713 isolates had His445 mutated than MDR isolates (27.8\% of RMR and $9.5 \%$ of MDR, $p$

$714<0.00001$ ), and mutations to amino acids Ser450 and Asp435 were more prevalent in

715 MDR isolates than RMR isolates $(43.7 \%$ of RMR and $65.8 \%$ of MDR $(p<0.00001)$,

716 and $9.3 \%$ of $\operatorname{RMR}$ and $15.5 \%$ of $\operatorname{MDR}(p=0.00328)$ respectively).

717

718 Relatively little difference in the mutational profiles for $r p o B$ mutations falling 719 outside the RRDR was found; many RMR mutations were also seen in MDR isolates 720 and those found exclusively in RMR were at $<2 \%$ prevalence (Fig. S2). The most 721 common non-RRDR mutation in both MDR and RMR isolates was a cytosine to 722 thymine mutation 61 bases upstream of the rpoB start codon $(10.1 \%$ and $8.6 \%$ of 723 isolates respectively). The resistance conferring mutations, V695L and V170F, were 724 seen at low proportions with no significant difference between MDR and RMR isolates 725 (V695L was seen in 0.5\% MDR isolates and $1.3 \%$ of RMR isolates, and V170F was seen in $0.8 \%$ of MDR isolates and $1.7 \%$ of RMR isolates). 
a

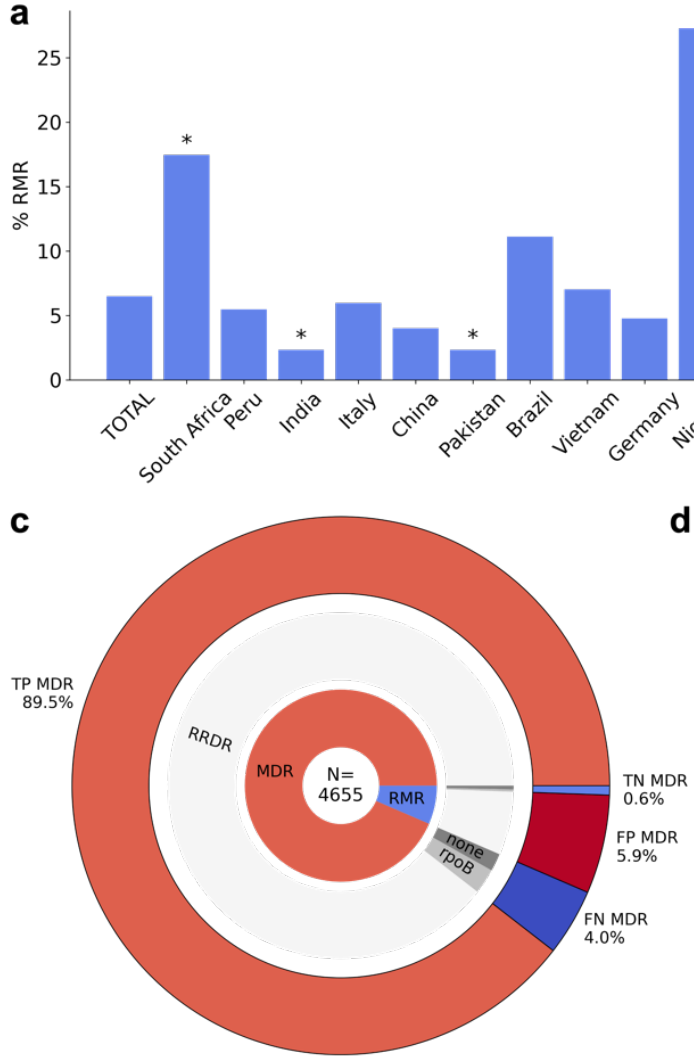

C

728

729

730 that mutation. b

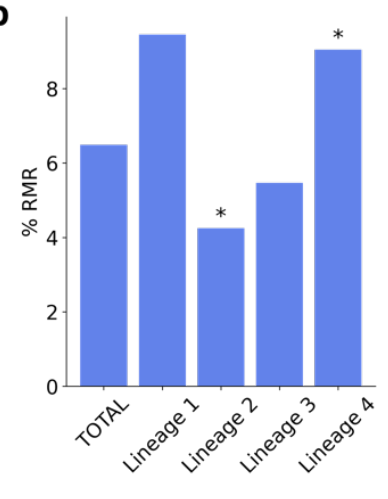

d

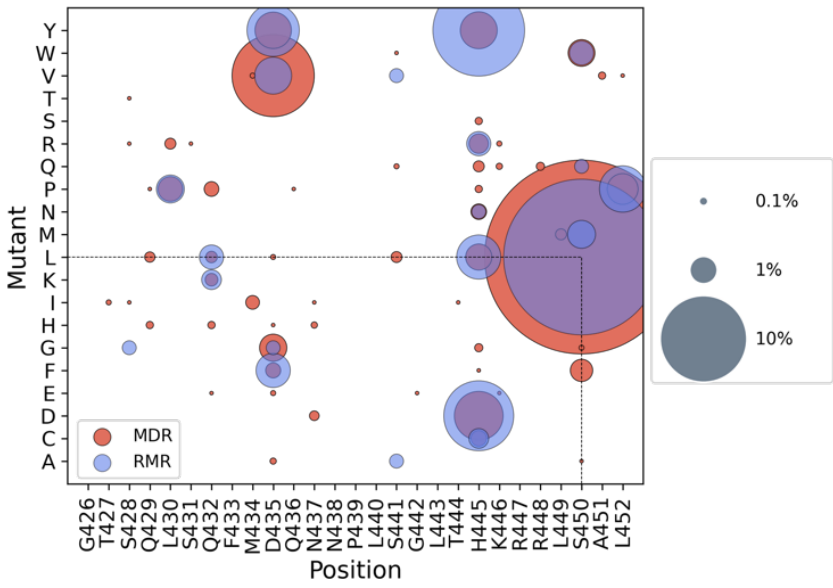

Figure 6: Rifampicin mono resistance a) Percentage of rifampicin resistant isolates that are rifampicin mono-resistant (RMR) by country of isolate origin. * indicates RMR proportions that were significantly different from that of the total dataset using a two tailed z-test with $95 \%$ confidence. b) RMR prevalence by lineage. * indicates RMR proportions that were significantly different from that of the total dataset using a two tailed z-test with $95 \%$ confidence. c) MDR predictions for rifampicin resistant isolates made using the Xpert $₫$ MTB/RIF assay proxy. N is the total number of rifampicin resistant isolates. The inner ring shows the proportion of rifampicin resistant isolates that are MDR and RMR. The middle ring represents the proportions of MDR and RMR isolates that have a SNP (synonymous or non-synonymous) in the RRDR of rpoB (RRDR), no RRDR SNP but a SNP elsewhere in the rpoB gene (rpoB) and no rpoB mutations (none). The outer ring shows the expected true positive (TP), true negative (TN), false positive (FP) and false negative (FN) MDR predictions of Xpert ${ }^{8}$ MTB/RIF assay, based on the SNPs present in the rifampicin resistant isolates. d) Non-synonymous mutations found in the RRDR of rpoB in RMR isolates and MDR isolates. Presence of a coloured spot indicates that the mutation was found in RMR/MDR isolates and spot size corresponds to the proportion of RMR or MDR isolates carrying 
Discussion

This compendium of $M$. tuberculosis clinical isolates is the result of an extensive global effort by the CRyPTIC consortium to better map the genetic variation associated with drug resistance. Through its sheer size and by oversampling for resistance, the compendium gives an unparalleled view of resistance patterns in the panel of 13 antitubercular compounds studied, providing more nuance to the traditional classification (nonMDR/MDR/XDR) nomenclature. These insights could be used to inform future treatment guidelines and steer the development of improved diagnostics.

For first-line drugs, molecular based diagnostic assays have vastly improved the detection of and the speed at which we find drug resistant TB cases, resulting in improved quality of care for patients. However, relying solely on these diagnostic methods has several drawbacks. Aside from the Xpert® MTB/RIF assay potentially increasing false positive MDR diagnoses as discussed earlier in the RMR case study, the assay assumes isoniazid resistance upon detection of rifampicin resistance. Thus, less is known about the prevalence of mono isoniazid resistance or 'true' cases of MDR (confirmed rifampicin and isoniazid resistance) (REFWHO). A greater amount of isoniazid-resistant and rifampicin-susceptible $(\mathrm{Hr}-\mathrm{TB})$ isolates were contributed by CRyPTIC countries than RMR isolates ( $n=1470$ versus $n=302$ ), a pattern also recently observed in a global prevalence study [50]. A modified 6-month treatment regimen is now recommended for $\mathrm{Hr}$-TB (rifampicin, ethambutol, levofloxacin and pyrazinamide), and as a result of inadequate diagnosis many of the 1.4 million global Hr-TB estimated cases would have received inadequate and unnecessarily longer

774 treatment regimens [1, 50]. Encouragingly, CRyPTIC isolates with a $\mathrm{Hr}-\mathrm{TB}$ background exhibited relatively low levels of resistance to other antitubercular drugs, 
including those in the augmented regimen (Fig. 4c). However, without the tools appropriate to assess and survey this, we will continue to misdiagnose and infectively treat these clinical cases.

In 2018, CRyPTIC and the 100,000 Genomes project demonstrated using WGS that genotypic prediction correlates well with culture-based phenotype for first-line drugs, which is reflected in our summary of the genetic catalogue applied to this specificity, there is still more to learn, as exemplified by the isolates in the compendium that despite being resistant to rifampicin and isoniazid could not be described genetically (Table 2). This shortfall, along with the limitations of molecular based diagnostic assays, highlights the need for continual genetic surveillance as drug resistant cases rise and shines a favourable light on a WGS-led approach.

One of the particular strengths of this compendium lies with the data collated for second-line drugs. A greater proportion of drug resistant isolates had additional resistance to fluoroquinolones than second line injectable drugs (Fig. 4a). This could be because of more widespread use of fluoroquinolones to treat bacterial infections in general as well as their ease of administration, hence being recommended over injectables for longer MDR treatment regimens [1]. Concerningly, we found that resistance to levofloxacin and moxifloxacin, and kanamycin and amikacin, were more common than resistance to the mycobacterial specific drug ethambutol in an isoniazid and rifampicin susceptible background (Fig. 4b) which suggests a level of pre-existing second line resistance. This concurs with a systematic review that found patients

800 previously prescribed fluoroquinolones were 3 times more likely to have 
801 fluoroquinolone resistant TB [51]. Careful stewardship of fluoroquinolones both in TB

802 and other infectious diseases will be paramount for the success of treatment regimens.

803 Despite the variability in sample collection, the observed high proportions of

804 fluoroquinolone resistant MDR/RR isolates from some countries also suggests that

805 MDR treatment regimens could be improved by optimisation on a geographic basis.

from each class; for example, the WHO recommends switching from kanamycin to amikacin when treating MDR TB patients [52]. The compendium supports such

810 recommendations as we saw more resistance to kanamycin than amikacin in not just

811 MDR/RR but also all other phenotypic backgrounds. For fluoroquinolones, more

812 isolates were resistant to levofloxacin than moxifloxacin in all phenotypic backgrounds

813 suggesting moxifloxacin may by the most appropriate fluoroquinolone to recommend.

814 However, the amenability of drugs to catalogue-based genetic diagnostics is also an

815 important consideration, and our data suggest levofloxacin resistance could be 816 predicted more reliably than moxifloxacin with fewer false positives predicted (Table 817 2).

Testing for fluoroquinolone resistance using molecular diagnostic tests remains

820 limited. However, the limited global data from the past 15 years suggests that the 821 proportion of MDR/RR TB cases resistant to fluoroquinolones sits at around $20 \%$, with 822 these cases primarily found in regions of high MDR-TB burden [1]. While tools such 823 as the Cepheid Xpert® MTB/XDR cartridge, which has just been approved for use by 824 the WHO, will permit both isoniazid and fluoroquinolone testing to be increased, the 825 same pitfalls are to be encountered regarding targeted diagnostic assays [53]. In 
contrast, the genetic survey in this study demonstrates the potential of WGS for

827 genetic prediction of resistance to second-line drugs and studies within the consortium

828 to investigate this are underway.

$830 \quad$ The CRyPTIC compendium has facilitated the first global survey of resistance

831 to NRDs. Reassuringly, prevalence of resistance to the NRDs was lower than for first-

832 and second-line agents in the dataset as a whole (Fig. 3a), and resistance to the new

833 drugs bedaquiline and delamanid was less common than the repurposed drugs

834 clofazimine and linezolid in an MDR/RR background (Fig. 4c). However, the presence

835 of higher levels of delamanid and clofazimine resistance than ethambutol resistance

836 in the isoniazid and rifampicin susceptible background does suggest some pre-existing

837 propensity towards NRD resistance (Fig. 4b).

838

Co-resistance between all two-drug combinations of NRDs was seen in isolates

840 in the compendium, the most common being isolates resistant to both bedaquiline and

841 clofazimine. This link is well documented and has been attributed to shared resistance

842 mechanisms such as non-synonymous mutations in rv0678 which were found in both

843 clofazimine and bedaquiline resistant isolates in the compendium [42] (Fig. 5b,c).

844 Increased clofazimine use could further increase the prevalence of $M$. tuberculosis

845 isolates with clofazimine and bedaquiline co-resistance, limiting MDR treatment

846 options such as using bedaquiline as the backbone of a shorter MDR regimen [54].

847 Therefore, proposed usage of clofazimine for other infectious diseases, including

848 COVID-19, should be carefully considered [55]. 
851 prevent the development of co-resistance, which could occur relatively quickly [56];

852 the rates of spontaneous evolution of delamanid resistance in vitro has been shown

853 to be comparable to that of isoniazid, and likewise bedaquiline resistance arises at a

854 comparable rate to rifampicin resistance [57]. In this compendium, $12.9 \%$ bedaquiline resistant isolates were resistant to delamanid and $7.1 \%$ delamanid resistant isolates were resistant to bedaquiline. Several scenarios could account for this, including the presence of shared resistance mechanisms. For example, as bedaquiline targets energy metabolism within the cell, changes to cope with energy/nutrient imbalances upon the acquisition of resistance-associated ATPase pump mutations may result in cross resistance to delamanid in a yet unknown or unexplored mechanism [58].

861 Additionally, off-target mutations in the $\mathrm{mmp} / 3$ gene (involved in export), are associated with resistance to delamanid, bedaquiline, clofazimine and linezolid, and were seen in isolates resistant to each of these drugs, although we note this could be artefactual (Fig. 5b-e). In any case, it is imperative that genetic determinants of resistance are fully explored for our last resort NRDs, with special attention given to those mechanisms that could be shared with other agents. In the meantime, careful stewardship and phenotypic and genotypic surveillance of the NRDs should be

868 implemented, including linezolid and clofazimine which are now group A and B drugs 869 respectively for MDR treatment [1].

872 consortium that make use of this compendium, including: i) relating genetic mutations

873 to quantitative changes in the minimum inhibitory concentrations of different drugs

874 [58]; ii) genome-wide association studies [59]; iii) training machine learning models 
875 that can predict resistance [60]; iv) exploration of the genetic determinants of

876 resistance to second line and NRDs [61]. Collectively these studies share the same

877 aim of facilitating the implementation of WGS-directed resistance prediction in the

878 clinic. Finally, we urge other researchers to explore this large dataset of $M$.

879 tuberculosis clinical isolates and hope it will lead to a wave of new and inciteful studies

880 that will positively serve the TB community for years to come.

881

882

883

884

885

886

Author contributions

887

D.W.C, T.E.A.P, S.H, A.L.G.C, A.W.S, T.M.W, P.W.F, D.M.C designed the CRyPTIC

study and all contributing laboratories collected samples and provided data. MIC data and genetic information was retrieved and processed by P.W.F, S.H, A.L.G.C, Z.I, M.H and J.K. A.B and K.M.M designed and performed all analyses for this manuscript. A.B and K.M.M wrote the manuscript with feedback from CRyPTIC partners.

\section{Acknowledgements}

895 We thank Faisal Masood Khanzada and Alamdar Hussain Rizvi (NTRL, Islamabad, 896 Pakistan), Angela Starks and James Posey (Centers for Disease Control and

897 Prevention, Atlanta, USA), and Juan Carlos Toro and Solomon Ghebremichael (Public

898 Health Agency of Sweden, Solna, Sweden), Iñaki Comas and Álvaro Chiner-Oms

899 (Instituto de Biología Integrativa de Sistemas, Valencia, Spain; CIBER en 
900 Epidemiología y Salud Pública, Valencia, Spain; Instituto de Biomedicina de Valencia,

901 IBV-CSIC, Valencia, Spain).

902

\section{$903 \quad$ Funding bodies}

904 This work was supported by Wellcome Trust/Newton Fund-MRC Collaborative Award 905 (200205/Z/15/Z); and Bill \& Melinda Gates Foundation Trust (OPP1133541). Oxford 906 CRyPTIC consortium members are funded/supported by the National Institute for

907 Health Research (NIHR) Oxford Biomedical Research Centre (BRC), the views 908 expressed are those of the authors and not necessarily those of the NHS, the NIHR 909 or the Department of Health, and the National Institute for Health Research (NIHR)

910 Health Protection Research Unit in Healthcare Associated Infections and Antimicrobial 911 Resistance, a partnership between Public Health England and the University of

912 Oxford, the views expressed are those of the authors and not necessarily those of the

913 NIHR, Public Health England or the Department of Health and Social Care. J.M. is

914 supported by the Wellcome Trust $(203919 / Z / 16 / Z)$. Z.Y. is supported by the National

915 Science and Technology Major Project, China Grant No. 2018ZX10103001. K.M.M. is

916 supported by EMBL's EIPOD3 programme funded by the European Union's Horizon

9172020 research and innovation programme under Marie Skłodowska Curie Actions.

918 T.C.R. is funded in part by funding from Unitaid Grant No. 2019-32-FIND MDR. R.S.O.

919 is supported by FAPESP Grant No. 17/16082-7. L.F. received financial support from

920 FAPESP Grant No. 2012/51756-5. B.Z. is supported by the National Natural Science

921 Foundation of China (81991534) and the Beijing Municipal Science \& Technology

922 Commission (Z201100005520041). N.T.T.T. is supported by the Wellcome Trust

923 International Intermediate Fellowship $(206724 / Z / 17 / Z)$. G.T. is funded by the

924 Wellcome Trust. R.W. is supported by the South African Medical Research Council. 
J.C. is supported by the Rhodes Trust and Stanford Medical Scientist Training

926 Program (T32 GM007365). A.L. is supported by the National Institute for Health

927 Research (NIHR) Health Protection Research Unit in Respiratory Infections at Imperial

928 College London. S.G.L. is supported by the Fonds de Recherche en Santé du Québec.

929 C.N. is funded by Wellcome Trust Grant No. 203583/Z/16/Z. A.V.R. is supported by

930 Research Foundation Flanders (FWO) under Grant No. G0F8316N (FWO Odysseus).

931 G.M. was supported by the Wellcome Trust (098316, 214321/Z/18/Z, and

932 203135/Z/16/Z), and the South African Research Chairs Initiative of the Department

933 of Science and Technology and National Research Foundation (NRF) of South Africa

934 (Grant No. 64787). The funders had no role in the study design, data collection, data

935 analysis, data interpretation, or writing of this report. The opinions, findings and

936 conclusions expressed in this manuscript reflect those of the authors alone. L.G. was

937 supported by the Wellcome Trust (201470/Z/16/Z), the National Institute of Allergy and

938 Infectious Diseases of the National Institutes of Health under award number

939 1R01Al146338, the GOSH Charity (VC0921) and the GOSH/ICH Biomedical

940 Research Centre (www.nihr.ac.uk). A.B. is funded by the NDM Prize Studentship from

941 the Oxford Medical Research Council Doctoral Training Partnership and the Nuffield

942 Department of Clinical Medicine. D.J.W. is supported by a Sir Henry Dale Fellowship

943 jointly funded by the Wellcome Trust and the Royal Society (Grant No. 101237/Z/13/B)

944 and by the Robertson Foundation. A.S.W. is an NIHR Senior Investigator. T.M.W. is a

945 Wellcome Trust Clinical Career Development Fellow (214560/Z/18/Z). A.S.L. is

946 supported by the Rhodes Trust. R.J.W. receives funding from the Francis Crick

947 Institute which is supported by Wellcome Trust, (FC0010218), UKRI (FC0010218),

948 and CRUK (FC0010218). T.C. has received grant funding and salary support from US

$949 \mathrm{NIH}, \mathrm{CDC}$, USAID and Bill and Melinda Gates Foundation. The computational aspects 
950 of this research were supported by the Wellcome Trust Core Award Grant Number

$951203141 / Z / 16 / Z$ and the NIHR Oxford BRC. Parts of the work were funded by the

952 German Center of Infection Research (DZIF). The Scottish Mycobacteria Reference

953 Laboratory is funded through National Services Scotland. The Wadsworth Center

954 contributions were supported in part by Cooperative Agreement No. U600E000103

955 funded by the Centers for Disease Control and Prevention through the Association of

956 Public Health Laboratories and NIH/NIAID grant Al-117312. Additional support for sequencing and analysis was contributed by the Wadsworth Center Applied Genomic

958 Technologies Core Facility and the Wadsworth Center Bioinformatics Core. SYNLAB

959 Holding Germany GmbH for its direct and indirect support of research activities in the

960 Institute of Microbiology and Laboratory Medicine Gauting. N.R. thanks the

961 Programme National de Lutte contre la Tuberculose de Madagascar.

962

\section{Wellcome Trust Open Access}

964 This research was funded in part, by the Wellcome Trust/Newton Fund-MRC

965 Collaborative Award [200205/Z/15/Z]. For the purpose of Open Access, the author has applied a CC BY public copyright licence to any Author Accepted Manuscript version arising from this submission.

968

969 This research was funded, in part, by the Wellcome Trust [214321/Z/18/Z, and $970203135 / Z / 16 / Z]$. For the purpose of open access, the author has applied a CC BY 971 public copyright licence to any Author Accepted Manuscript version arising from this 972 submission.

\section{Competing Interest}


977 E.R. is employed by Public Health England and holds an honorary contract with

978 Imperial College London. I.F.L. is Director of the Scottish Mycobacteria Reference

979 Laboratory. S.N. receives funding from German Center for Infection Research,

980 Excellenz Cluster Precision Medicine in Chronic Inflammation, Leibniz Science

981 Campus Evolutionary Medicine of the LUNG (EvoLUNG)tion EXC 2167. P.S. is a 982 consultant at Genoscreen. T.R. is funded by NIH and DoD and receives salary support

983 from the non-profit organization FIND. T.R. is a co-founder, board member and

984 shareholder of Verus Diagnostics Inc, a company that was founded with the intent of

985 developing diagnostic assays. Verus Diagnostics was not involved in any way with

986 data collection, analysis or publication of the results. T.R. has not received any

987 financial support from Verus Diagnostics. UCSD Conflict of Interest office has

988 reviewed and approved T.R.'s role in Verus Diagnostics Inc. T.R. is a co-inventor of a

989 provisional patent for a TB diagnostic assay (provisional patent \#: 63/048.989). T.R. is

990 a co-inventor on a patent associated with the processing of TB sequencing data

991 (European Patent Application No. 14840432.0 \& USSN 14/912,918). T.R. has agreed

992 to "donate all present and future interest in and rights to royalties from this patent" to

993 UCSD to ensure that he does not receive any financial benefits from this patent. S.S.

994 is working and holding ESOPs at HaystackAnalytics Pvt. Ltd. (Product: Using whole

995 genome sequencing for drug susceptibility testing for Mycobacterium tuberculosis).

996 G.F.G. is listed as an inventor on patent applications for RBD-dimer-based CoV

997 vaccines. The patents for RBD-dimers as protein subunit vaccines for SARS-CoV-2

998 have been licensed to Anhui Zhifei Longcom Biopharmaceutical Co. Ltd, China.

999

1000

1001 


\section{Supplemental}

a
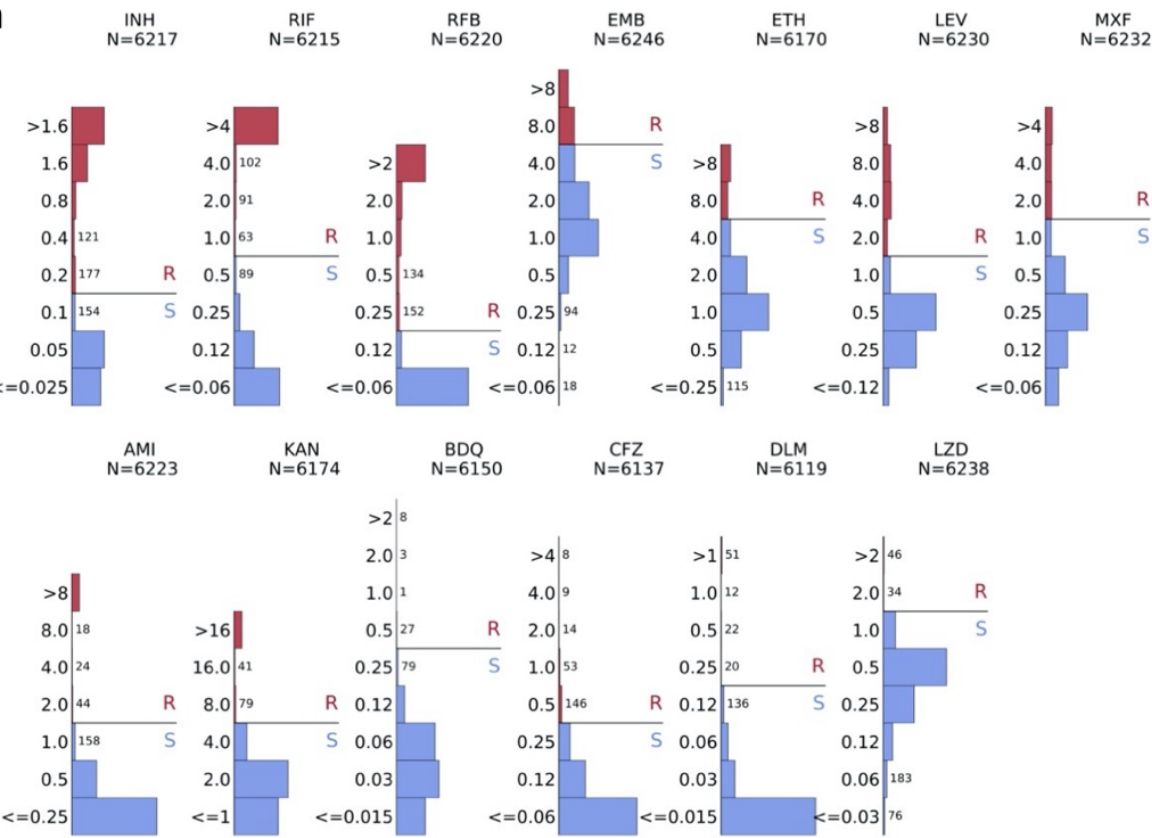

b
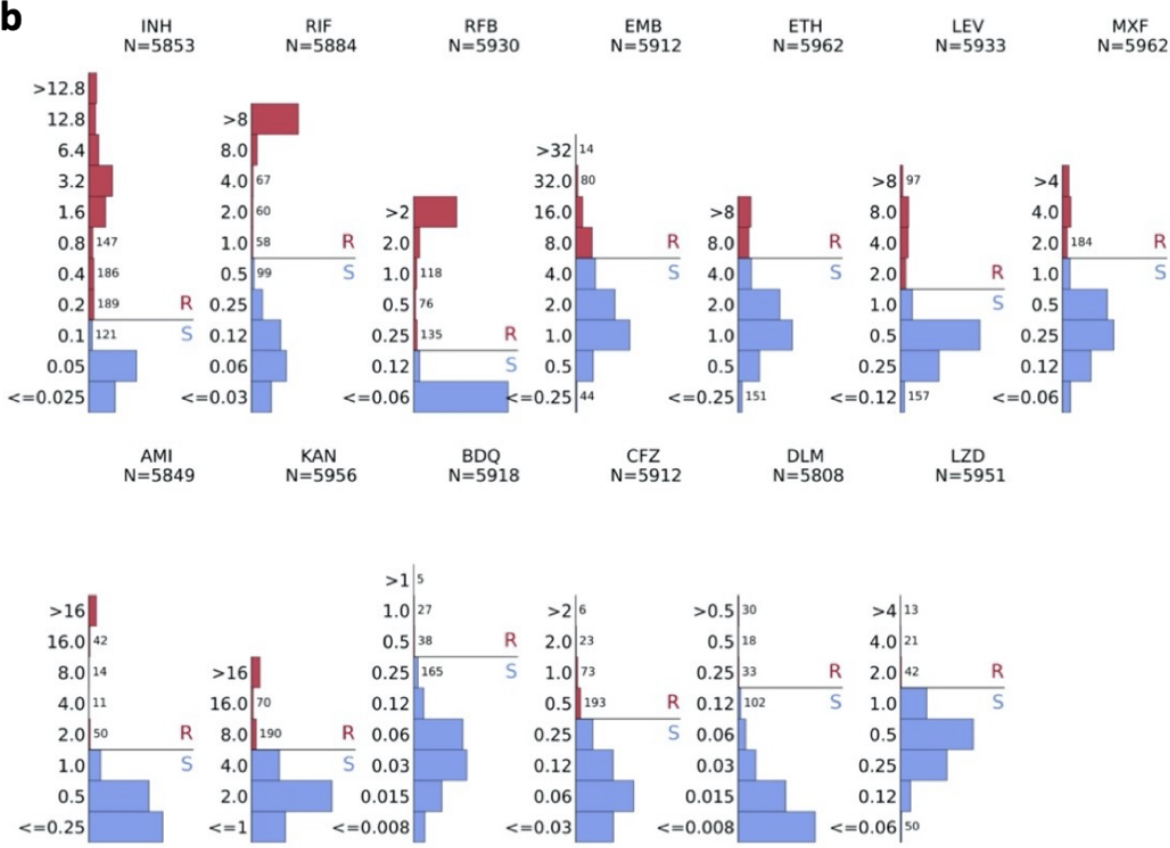

1004 Figure S1: Per drug MIC distributions of isolates plated on CRyPTIC designed variations on the Thermo Fischer Sensititre MYCOTB MIC plate; UKMYC5 (a) and UKMYC6 (b). The solid black line represents the ecological resistance cut-off (ECOFF) for each drug as determined by [11]. Isolates with an MIC above the cut-off are considered resistant. $\mathrm{N}$ denotes the total number of isolates tested on each plate that returned a phenotype for each drug. 


\begin{tabular}{|c|c|c|}
\hline UNIQUEID & $\begin{array}{l}\text { COUNTRY OF } \\
\text { ORIGIN }\end{array}$ & LINEAGE \\
\hline $\begin{array}{c}\text { site.11.subj.XTB-18-224.lab.XTB-18- } \\
\text { 224.iso.1 }\end{array}$ & UNKNOWN & Lineage 2 \\
\hline $\begin{array}{c}\text { site.10.subj.YA00026182.lab.YA00026182.i } \\
\text { so.1 }\end{array}$ & ZAF & Lineage 4 \\
\hline
\end{tabular}

1012 Table S1: Sample information for isolates classified as resistant to all 13

1013 CRyPTIC drugs tested. The country of origin is specified using the 3-letter country codes 1014 (alpha-3) defined by ISO 3166-1.

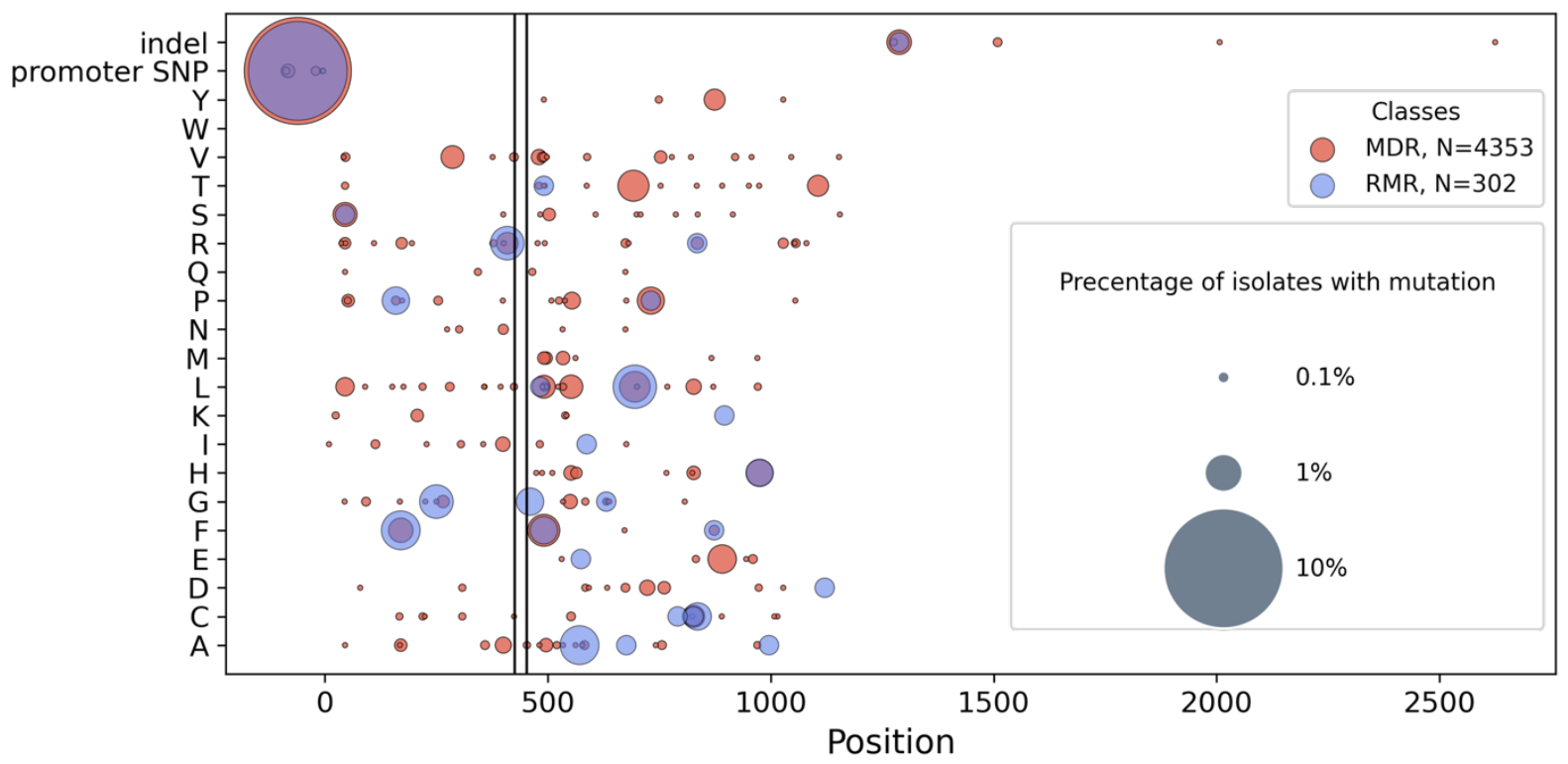

1016 Figure S2: Non-synonymous mutations found outside the RRDR of rpoB in RMR

1017 isolates and MDR isolates. Presence of a coloured spot indicates that the mutation was 1018 found in RMR/MDR isolates and spot size corresponds to the proportion of RMR or MDR isolates 1019 carrying that mutation. 


\begin{tabular}{|c|c|c|c|c|c|c|c|}
\hline & $\begin{array}{c}\text { Animal/ } \\
\text { other }\end{array}$ & L1 & L2 & L3 & L4 & L6 & Total \\
\hline Albania & 0 & 0 & 0 & 0 & 7 & 0 & 7 \\
\hline Algeria & 0 & 0 & 0 & 0 & 25 & 0 & 25 \\
\hline Brazil & 1 & 1 & 8 & 0 & 335 & 0 & 345 \\
\hline Burkina Faso & 0 & 3 & 1 & 8 & 65 & 1 & 78 \\
\hline China & 7 & 2 & 722 & 16 & 374 & 0 & 1121 \\
\hline Germany & 1 & 25 & 235 & 61 & 403 & 1 & 726 \\
\hline India & 11 & 676 & 1573 & 1184 & 560 & 0 & 4004 \\
\hline Italy & 11 & 38 & 192 & 113 & 866 & 4 & 1224 \\
\hline Japan & 0 & 0 & 0 & 0 & 1 & 0 & 1 \\
\hline Kyrgyzstan & 0 & 0 & 25 & 0 & 3 & 0 & 28 \\
\hline Nepal & 0 & 5 & 137 & 34 & 21 & 0 & 197 \\
\hline Nigeria & 0 & 0 & 0 & 0 & 15 & 0 & 15 \\
\hline Pakistan & 1 & 30 & 23 & 363 & 74 & 0 & 491 \\
\hline Peru & 2 & 1 & 360 & 0 & 2328 & 0 & 2691 \\
\hline South Africa & 1 & 61 & 874 & 57 & 1162 & 0 & 2155 \\
\hline Sweden & 0 & 7 & 280 & 6 & 134 & 0 & 427 \\
\hline Taiwan & 0 & 18 & 149 & 0 & 44 & 0 & 211 \\
\hline Tajikistan & 0 & 0 & 15 & 0 & 4 & 0 & 19 \\
\hline Tunisia & 0 & 0 & 0 & 0 & 2 & 0 & 2 \\
\hline Turkmenistan & 0 & 0 & 88 & 1 & 30 & 0 & 119 \\
\hline Ukraine & 0 & 0 & 21 & 0 & 9 & 0 & 30 \\
\hline UK & 0 & 0 & 3 & 3 & 1 & 0 & 7 \\
\hline Vietnam & 0 & 283 & 892 & 4 & 109 & 0 & 1288 \\
\hline Total & 35 & 1150 & 5598 & 1850 & 6572 & 6 & 15211 \\
\hline
\end{tabular}

1026 Table S2: Lineages - $v$ - geographical location of origin/contribution for CRyPTIC 


\begin{tabular}{|c|c|c|c|c|c|c|c|c|c|c|c|c|c|c|c|c|c|c|c|c|c|c|c|}
\hline 1 & 0 & 0 & 0 & 0 & 0 & 0 & 1 & 0 & 0 & 0 & 0 & 0 & 0 & 0 & 0 & 0 & 0 & 0 & 0 & 0 & 0 & 0 & 0 \\
\hline 1.1.1 & 0 & 0 & 0 & 2 & 0 & 0 & 1 & 2 & 0 & 0 & 0 & 0 & 0 & 0 & 0 & 0 & 0 & 0 & 0 & 0 & 0 & 0 & 38 \\
\hline 1.1.1.1 & 0 & 0 & 0 & 0 & 0 & 2 & 0 & 0 & 0 & 0 & 0 & 0 & 0 & 0 & 0 & 0 & 0 & 0 & 0 & 0 & 0 & 0 & 188 \\
\hline 1.1 .2 & 0 & 0 & 0 & 1 & 1 & 12 & 316 & 16 & 0 & 0 & 2 & 0 & 23 & 0 & 0 & 1 & 0 & 0 & 0 & 0 & 0 & 0 & 0 \\
\hline 1.1.3 & 0 & 0 & 1 & 0 & 0 & 1 & 52 & 4 & 0 & 0 & 1 & 0 & 1 & 0 & 6 & 0 & 0 & 0 & 0 & 0 & 0 & 0 & 2 \\
\hline 1.2 .1 & 0 & 0 & 0 & 0 & 0 & 8 & 12 & 11 & 0 & 0 & 0 & 0 & 0 & 1 & 0 & 3 & 16 & 0 & 0 & 0 & 0 & 0 & 24 \\
\hline 1.2 .2 & 0 & 0 & 0 & 0 & 1 & 2 & 259 & 5 & 0 & 0 & 2 & 0 & 4 & 0 & 54 & 3 & 1 & 0 & 0 & 0 & 0 & 0 & 1 \\
\hline 2 & 0 & 0 & 0 & 0 & 0 & 1 & 0 & 0 & 0 & 0 & 0 & 0 & 0 & 0 & 0 & 0 & 0 & 0 & 0 & 0 & 0 & 0 & 0 \\
\hline 2.1 & 0 & 0 & 0 & 0 & 1 & 1 & 0 & 0 & 0 & 0 & 0 & 0 & 0 & 1 & 0 & 0 & 20 & 0 & 0 & 0 & 0 & 0 & 34 \\
\hline 2.2 & 0 & 0 & 5 & 1 & 284 & 19 & 272 & 14 & 0 & 0 & 68 & 0 & 6 & 231 & 181 & 13 & 56 & 0 & 0 & 0 & 0 & 0 & 271 \\
\hline 2.2.1 & 0 & 0 & 0 & 0 & 37 & 4 & 2 & 2 & 0 & 0 & 0 & 0 & 0 & 3 & 224 & 1 & 21 & 0 & 0 & 0 & 0 & 0 & 26 \\
\hline 2.2.10 & 0 & 0 & 0 & 0 & 1 & 117 & 1 & 84 & 0 & 16 & 0 & 0 & 3 & 0 & 0 & 70 & 0 & 5 & 0 & 75 & 9 & 1 & 0 \\
\hline 2.2.2 & 0 & 0 & 0 & 0 & 32 & 8 & 5 & 11 & 0 & 1 & 0 & 0 & 1 & 0 & 0 & 1 & 5 & 0 & 0 & 0 & 1 & 0 & 8 \\
\hline 2.2 .3 & 0 & 0 & 2 & 0 & 172 & 3 & 290 & 4 & 0 & 0 & 21 & 0 & 2 & 1 & 3 & 1 & 10 & 0 & 0 & 0 & 0 & 0 & 100 \\
\hline 2.2.4 & 0 & 0 & 0 & 0 & 3 & 3 & 4 & 8 & 0 & 0 & 0 & 0 & 0 & 0 & 37 & 1 & 1 & 0 & 0 & 0 & 0 & 0 & 70 \\
\hline 2.2 .5 & 0 & 0 & 0 & 0 & 59 & 1 & 12 & 5 & 0 & 0 & 0 & 0 & 2 & 84 & 1 & 1 & 13 & 0 & 0 & 0 & 0 & 0 & 45 \\
\hline 2.2.6 & 0 & 0 & 1 & 0 & 22 & 8 & 226 & 2 & 0 & 0 & 9 & 0 & 7 & 4 & 204 & 1 & 4 & 0 & 0 & 0 & 0 & 0 & 154 \\
\hline 2.2.7 & 0 & 0 & 0 & 0 & 97 & 4 & 691 & 5 & 0 & 1 & 38 & 0 & 0 & 20 & 206 & 5 & 18 & 0 & 0 & 0 & 0 & 0 & 164 \\
\hline 2.2.8 & 0 & 0 & 0 & 0 & 4 & 0 & 42 & 0 & 0 & 0 & 1 & 0 & 2 & 0 & 0 & 0 & 0 & 0 & 0 & 0 & 0 & 0 & 2 \\
\hline 2.2 .9 & 0 & 0 & 0 & 0 & 0 & 64 & 0 & 51 & 0 & 6 & 0 & 0 & 0 & 2 & 0 & 174 & 0 & 10 & 0 & 11 & 11 & 2 & 0 \\
\hline 3 & 0 & 0 & 0 & 8 & 15 & 37 & 828 & 81 & 0 & 0 & 28 & 0 & 346 & 0 & 34 & 5 & 0 & 0 & 0 & 1 & 0 & 2 & 2 \\
\hline 3.1.1 & 0 & 0 & 0 & 0 & 0 & 17 & 17 & 25 & 0 & 0 & 1 & 0 & 0 & 0 & 19 & 1 & 0 & 0 & 0 & 0 & 0 & 1 & 2 \\
\hline 3.1.2 & 0 & 0 & 0 & 0 & 1 & 5 & 201 & 3 & 0 & 0 & 1 & 0 & 7 & 0 & 2 & 0 & 0 & 0 & 0 & 0 & 0 & 0 & 0 \\
\hline 3.1.2.1 & 0 & 0 & 0 & 0 & 0 & 2 & 119 & 3 & 0 & 0 & 4 & 0 & 6 & 0 & 0 & 0 & 0 & 0 & 0 & 0 & 0 & 0 & 0 \\
\hline 4 & 0 & 0 & 1 & 0 & 6 & 4 & 0 & 6 & 0 & 0 & 0 & 0 & 0 & 100 & 1 & 1 & 0 & 0 & 0 & 1 & 0 & 0 & 0 \\
\hline 4.1 & 0 & 0 & 0 & 1 & 0 & 1 & 0 & 2 & 0 & 0 & 0 & 0 & 0 & 0 & 0 & 0 & 0 & 0 & 0 & 0 & 0 & 0 & 0 \\
\hline 4.1.1 & 0 & 0 & 0 & 0 & 0 & 6 & 5 & 10 & 0 & 0 & 1 & 0 & 0 & 154 & 13 & 1 & 0 & 0 & 0 & 0 & 0 & 0 & 0 \\
\hline 4.1.1.1 & 0 & 0 & 5 & 0 & 1 & 3 & 20 & 5 & 0 & 0 & 1 & 0 & 2 & 5 & 54 & 1 & 0 & 0 & 0 & 0 & 0 & 0 & 1 \\
\hline 4.1.1.2 & 0 & 0 & 0 & 0 & 0 & 0 & 4 & 1 & 0 & 0 & 0 & 0 & 0 & 0 & 6 & 0 & 0 & 0 & 0 & 0 & 0 & 0 & 0 \\
\hline 4.1.1.3 & 0 & 0 & 1 & 6 & 1 & 0 & 30 & 7 & 1 & 0 & 3 & 0 & 2 & 68 & 138 & 0 & 0 & 0 & 0 & 0 & 0 & 0 & 1 \\
\hline 4.1.2 & 0 & 0 & 13 & 0 & 0 & 15 & 3 & 26 & 0 & 1 & 0 & 0 & 0 & 31 & 43 & 0 & 0 & 0 & 0 & 0 & 0 & 0 & 10 \\
\hline 4.1.2.1 & 1 & 8 & 87 & 10 & 0 & 111 & 56 & 183 & 0 & 0 & 3 & 2 & 4 & 653 & 54 & 5 & 0 & 1 & 0 & 0 & 1 & 0 & 17 \\
\hline 4.1.3 & 0 & 0 & 0 & 5 & 0 & 0 & 0 & 6 & 0 & 0 & 0 & 1 & 0 & 0 & 0 & 0 & 0 & 0 & 0 & 0 & 0 & 0 & 0 \\
\hline 4.10 & 4 & 6 & 35 & 5 & 1 & 110 & 166 & 207 & 0 & 1 & 3 & 0 & 28 & 128 & 170 & 4 & 5 & 2 & 1 & 6 & 2 & 0 & 12 \\
\hline 4.2 & 0 & 0 & 0 & 0 & 3 & 1 & 0 & 1 & 0 & 0 & 0 & 0 & 0 & 0 & 0 & 0 & 0 & 0 & 0 & 0 & 0 & 0 & 0 \\
\hline 4.2.1 & 0 & 0 & 0 & 0 & 0 & 9 & 3 & 46 & 0 & 0 & 1 & 0 & 2 & 0 & 2 & 4 & 0 & 0 & 0 & 8 & 3 & 1 & 0 \\
\hline 4.2.2 & 0 & 1 & 0 & 0 & 53 & 31 & 76 & 51 & 0 & 0 & 5 & 0 & 12 & 2 & 10 & 10 & 1 & 0 & 0 & 0 & 0 & 0 & 11 \\
\hline 4.2.2.1 & 0 & 0 & 0 & 0 & 0 & 5 & 0 & 9 & 0 & 0 & 0 & 0 & 0 & 0 & 0 & 0 & 0 & 0 & 0 & 0 & 0 & 0 & 0 \\
\hline 4.3 & 0 & 0 & 17 & 0 & 0 & 0 & 0 & 1 & 0 & 0 & 0 & 0 & 0 & 96 & 1 & 0 & 0 & 0 & 0 & 0 & 0 & 0 & 0 \\
\hline 4.3.1 & 0 & 3 & 0 & 1 & 0 & 1 & 2 & 21 & 0 & 0 & 0 & 0 & 0 & 8 & 0 & 1 & 0 & 0 & 0 & 0 & 0 & 0 & 1 \\
\hline 4.3.2 & 0 & 1 & 10 & 0 & 0 & 3 & 1 & 8 & 0 & 0 & 0 & 0 & 0 & 245 & 21 & 0 & 0 & 0 & 0 & 0 & 0 & 0 & 0 \\
\hline 4.3.2.1 & 0 & 0 & 0 & 0 & 0 & 0 & 0 & 0 & 0 & 0 & 0 & 0 & 0 & 0 & 234 & 0 & 0 & 0 & 0 & 0 & 0 & 0 & 0 \\
\hline 4.3.3 & 1 & 4 & 34 & 2 & 0 & 33 & 99 & 81 & 0 & 1 & 1 & 0 & 0 & 576 & 120 & 102 & 2 & 1 & 1 & 11 & 2 & 0 & 1 \\
\hline 4.3.4 & 0 & 0 & 1 & 0 & 0 & 0 & 8 & 0 & 0 & 0 & 0 & 0 & 0 & 1 & 0 & 0 & 0 & 0 & 0 & 0 & 0 & 0 & 0 \\
\hline 4.3.4.1 & 0 & 0 & 70 & 0 & 0 & 2 & 6 & 3 & 0 & 0 & 0 & 0 & 0 & 60 & 22 & 2 & 1 & 0 & 0 & 0 & 0 & 0 & 0 \\
\hline 4.3.4.2 & 0 & 0 & 38 & 0 & 0 & 6 & 7 & 27 & 0 & 0 & 0 & 0 & 0 & 132 & 12 & 0 & 0 & 0 & 0 & 0 & 1 & 0 & 0 \\
\hline 4.3.4.2.1 & 0 & 0 & 0 & 0 & 0 & 2 & 1 & 1 & 0 & 0 & 0 & 0 & 0 & 0 & 75 & 0 & 0 & 0 & 0 & 0 & 0 & 0 & 0 \\
\hline 4.4 & 0 & 0 & 0 & 0 & 0 & 0 & 13 & 0 & 0 & 0 & 0 & 0 & 0 & 1 & 0 & 0 & 0 & 0 & 0 & 0 & 0 & 0 & 0 \\
\hline 4.4.1 & 0 & 0 & 10 & 0 & 0 & 0 & 0 & 2 & 0 & 0 & 0 & 0 & 0 & 11 & 0 & 0 & 0 & 0 & 0 & 0 & 0 & 0 & 0 \\
\hline 4.4.1.1 & 0 & 0 & 4 & 0 & 0 & 16 & 4 & 66 & 0 & 0 & 1 & 0 & 0 & 22 & 180 & 2 & 0 & 0 & 0 & 0 & 0 & 0 & 4 \\
\hline 4.4.1.2 & 0 & 0 & 0 & 0 & 0 & 1 & 25 & 3 & 0 & 0 & 0 & 0 & 0 & 2 & 0 & 0 & 0 & 0 & 0 & 0 & 0 & 0 & 3 \\
\hline 4.4.2 & 0 & 0 & 0 & 0 & 124 & 1 & 4 & 1 & 0 & 0 & 0 & 0 & 0 & 4 & 0 & 0 & 5 & 0 & 0 & 0 & 0 & 0 & 16 \\
\hline 4.5 & 0 & 0 & 0 & 0 & 179 & 28 & 15 & 14 & 0 & 0 & 1 & 0 & 18 & 5 & 1 & 0 & 30 & 0 & 0 & 3 & 0 & 0 & 29 \\
\hline 4.6 & 0 & 1 & 0 & 1 & 0 & 1 & 1 & 7 & 0 & 0 & 0 & 2 & 0 & 0 & 0 & 0 & 0 & 0 & 0 & 0 & 0 & 0 & 0 \\
\hline 4.6.1.1 & 0 & 0 & 0 & 0 & 0 & 1 & 0 & 0 & 0 & 0 & 0 & 0 & 0 & 0 & 0 & 0 & 0 & 0 & 0 & 0 & 0 & 0 & 0 \\
\hline 4.6.1.2 & 0 & 0 & 0 & 0 & 0 & 1 & 0 & 0 & 0 & 0 & 0 & 0 & 0 & 0 & 0 & 1 & 0 & 0 & 0 & 0 & 0 & 0 & 0 \\
\hline 4.6.2 & 0 & 0 & 0 & 0 & 0 & 2 & 2 & 14 & 0 & 0 & 0 & 0 & 4 & 0 & 0 & 0 & 0 & 0 & 0 & 0 & 0 & 0 & 0 \\
\hline 4.6.2.1 & 0 & 0 & 0 & 0 & 0 & 0 & 1 & 0 & 0 & 0 & 1 & 0 & 1 & 0 & 0 & 0 & 0 & 0 & 0 & 0 & 0 & 0 & 0 \\
\hline 4.6.2.2 & 1 & 0 & 0 & 34 & 0 & 8 & 1 & 55 & 0 & 0 & 0 & 10 & 1 & 0 & 1 & 0 & 0 & 0 & 0 & 0 & 0 & 0 & 3 \\
\hline 6 & 0 & 0 & 0 & 1 & 0 & 1 & 0 & 4 & 0 & 0 & 0 & 0 & 0 & 0 & 0 & 0 & 0 & 0 & 0 & 0 & 0 & 0 & 0 \\
\hline
\end{tabular}




\begin{tabular}{l|lllllllllllllllllllllllllll} 
Bovis & 0 & 0 & 0 & 0 & 0 & 1 & 1 & 10 & 0 & 0 & 0 & 0 & 0 & 2 & 1 & 0 & 0 & 0 & 0 & 0 & 0 & 0 & 0 \\
Caprae & 0 & 0 & 0 & 0 & 0 & 0 & 0 & 1 & 0 & 0 & 0 & 0 & 0 & 0 & 0 & 0 & 0 & 0 & 0 & 0 & 0 & 0 & 0 \\
Mixed & 0 & 1 & 9 & 0 & 16 & 3 & 89 & 9 & 0 & 1 & 0 & 0 & 6 & 38 & 25 & 12 & 2 & 0 & 0 & 3 & 0 & 0 & 48 \\
Unknown & 0 & 0 & 1 & 0 & 7 & 0 & 0 & 0 & 0 & 0 & 0 & 0 & 1 & 0 & 0 & 0 & 0 & 0 & 0 & 0 & 0 & 0 & 0
\end{tabular}

1035 Table S3: Sub-lineages -v- geographical location of origin/contribution for 1036 CRyPTIC isolates

1037

1038

1039 in the CRyPTIC data. Pearson's chi-squared test, $\mathrm{X}$-squared $=7935.2, \mathrm{df}=110, p<2.2 \mathrm{e}-16$. The correlation plot indicates the relative contribution of each row-column pairing to the chisquare test score $(\%)$.

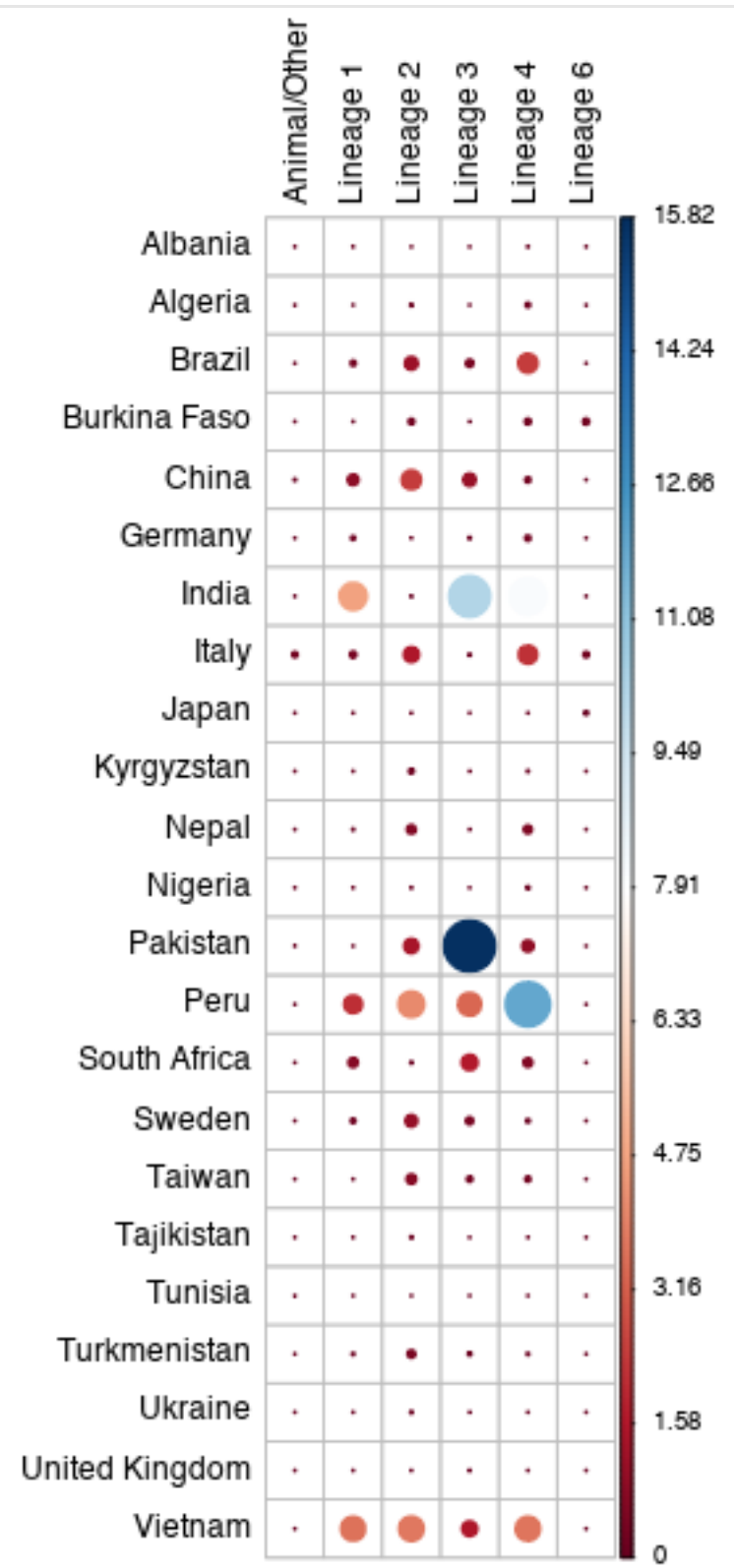




\begin{tabular}{r|lllllllllllll}
\multicolumn{1}{c}{} & INH & RIF & EMB & LEV & MXF & AMI & KAN & BDQ & CFZ & DLM & LZD & ETH & RFB \\
\hline INH & 100.0 & 74.8 & 38.0 & 34.0 & 27.8 & 14.0 & 17.0 & 1.5 & 5.5 & 1.7 & 2.0 & 28.2 & 70.4 \\
RIF & 93.5 & 100.0 & 46.3 & 41.4 & 34.1 & 17.2 & 20.8 & 1.8 & 6.0 & 1.7 & 2.3 & 29.4 & 91.3 \\
EMB & 98.5 & 95.9 & 100.0 & 53.9 & 47.1 & 23.3 & 26.4 & 2.4 & 8.2 & 2.3 & 3.7 & 35.8 & 87.3 \\
LEV & 93.3 & 90.2 & 56.9 & 100.0 & 78.5 & 27.3 & 31.3 & 3.1 & 9.4 & 2.9 & 4.7 & 39.2 & 87.1 \\
MXF & 95.0 & 92.3 & 61.9 & 97.6 & 100.0 & 29.9 & 34.6 & 3.2 & 9.9 & 2.7 & 5.3 & 41.9 & 88.8 \\
AMI & 93.0 & 90.4 & 58.9 & 65.3 & 57.8 & 100.0 & 90.4 & 2.4 & 13.0 & 5.2 & 8.1 & 40.7 & 82.8 \\
KAN & 89.4 & 86.7 & 53.0 & 59.5 & 53.2 & 72.0 & 100.0 & 1.8 & 10.6 & 3.2 & 5.7 & 40.4 & 80.0 \\
BDQ & 79.4 & 77.8 & 49.5 & 60.7 & 49.5 & 20.0 & 18.9 & 100.0 & 52.4 & 12.9 & 14.0 & 34.9 & 76.9 \\
CFZ & 61.9 & 52.8 & 34.9 & 38.2 & 32.1 & 21.8 & 22.5 & 10.6 & 100.0 & 9.4 & 10.2 & 27.0 & 53.7 \\
DLM & 55.2 & 44.4 & 28.2 & 33.2 & 23.9 & 24.4 & 19.1 & 7.1 & 26.3 & 100.0 & 19.3 & 22.1 & 45.4 \\
LZD & 77.6 & 69.9 & 53.2 & 64.7 & 58.7 & 46.7 & 41.2 & 9.8 & 34.2 & 24.1 & 100.0 & 42.6 & 66.7 \\
ETH & 96.5 & 79.4 & 47.0 & 48.7 & 41.9 & 21.1 & 26.2 & 2.2 & 8.3 & 2.4 & 3.9 & 100.0 & 77.1 \\
RFB & 93.3 & 96.8 & 44.5 & 42.1 & 34.4 & 16.6 & 20.3 & 1.9 & 6.4 & 1.9 & 2.3 & 29.9 & 100.0
\end{tabular}

1048 Table S4: Co-occurrence of antibiotic resistance in CRyPTIC M. tuberculosis

1049

1050

1051

1052

1053

1054

1055

1056

1057

1058

1059

1060

1061

1062

1063

1064

1065

1066

1067

1068

1069

1070

1071

1072

1073

1074

1075

1076

1077

isolates. The probability (\%) of an isolate being resistant to Drug 2 (top) if it is resistant to Drug 1 (left). Drug acronyms: INH = isoniazid, RIF = rifampicin, EMB = ethambutol, LEV = levofloxacin, $\mathrm{MXF}=$ moxifloxacin, $\mathrm{AMI}=$ amikacin, $\mathrm{KAN}=$ kanamycin, $\mathrm{BDQ}=$ bedaquiline, $\mathrm{CFZ}=$ clofazimine, $\mathrm{DLM}=$ delamanid, $\mathrm{LZD}=$ linezolid, $\mathrm{ETH}=$ ethionamide, $\mathrm{RFB}=$ rifabutin.

\section{References}

1. WHO, World Health Organisation: Global Tuberculosis Report 2020. 2020.

2. Boehme, C.C., et al., Feasibility, diagnostic accuracy, and effectiveness of decentralised use of the Xpert MTB/RIF test for diagnosis of tuberculosis and multidrug resistance: a multicentre implementation study. Lancet, 2011. 377(9776): p. 1495-505.

3. Shinnick, T.M., et al., Evaluation of the Cepheid Xpert MTB/RIF assay. Expert Rev Mol Diagn, 2015. 15(1): p. 9-22.

4. $\quad$ Sanchez-Padilla, E., et al., Detection of Drug-Resistant Tuberculosis by Xpert MTB/RIF in Swaziland. New England Journal of Medicine, 2015. 372(12): p. 11811182.

5. $\quad$ Beckert, P., et al., MDR M. tuberculosis outbreak clone in Eswatini missed by Xpert has elevated bedaquiline resistance dated to the pre-treatment era. Genome Medicine, 2020. 12(1): p. 104.

6. Makhado, N.A., et al., Outbreak of multidrug-resistant tuberculosis in South Africa undetected by WHO-endorsed commercial tests: an observational study. Lancet Infect Dis, 2018. 18(12): p. 1350-1359.

7. Consortium, T.C., et al., Prediction of Susceptibility to First-Line Tuberculosis Drugs by DNA Sequencing. N Engl J Med, 2018. 379(15): p. 1403-1415.

8. Pankhurst, L.J., et al., Rapid, comprehensive, and affordable mycobacterial diagnosis with whole-genome sequencing: a prospective study. Lancet Respir Med, 2016. 4(1): p. 49-58. 
9. $\quad$ Kalokhe, A.S., et al., Multidrug-resistant tuberculosis drug susceptibility and molecular diagnostic testing. Am J Med Sci, 2013. 345(2): p. 143-8.

10. Rancoita, P.M.V., et al., Validating a 14-Drug Microtiter Plate Containing Bedaquiline and Delamanid for Large-Scale Research Susceptibility Testing of Mycobacterium tuberculosis. Antimicrob Agents Chemother, 2018. 62(9).

11. Consortium, T.C. and P.W. Fowler, Epidemiological cutoff values for a 96-well broth microdilution plate for high-throughput research antibiotic susceptibility testing of $M$. tuberculosis. medRxiv, 2021: p. 2021.02.24.21252386.

12. Fowler, P.W., et al., Automated detection of bacterial growth on 96-well plates for high-throughput drug susceptibility testing of Mycobacterium tuberculosis.

Microbiology (Reading), 2018. 164(12): p. 1522-1530.

13. Fowler, P.W., et al., BashTheBug: a crowd of volunteers reproducibly and accurately measure the minimum inhibitory concentrations of 13 antitubercular drugs from photographs of 96-well broth microdilution plates. bioRxiv, 2021: $\mathrm{p}$. 2021.07.20.453060.

14. Bolger, A.M., M. Lohse, and B. Usadel, Trimmomatic: a flexible trimmer for Illumina sequence data. Bioinformatics, 2014. 30(15): p. 2114-20.

15. Li, H. and R. Durbin, Fast and accurate short read alignment with Burrows-Wheeler transform. Bioinformatics, 2009. 25(14): p. 1754-60.

16. Li, H., et al., The Sequence Alignment/Map format and SAMtools. Bioinformatics, 2009. 25(16): p. 2078-9.

17. Iqbal, Z., et al., De novo assembly and genotyping of variants using colored de Bruijn graphs. Nat Genet, 2012. 44(2): p. 226-32.

18. Hunt, M., et al., Antibiotic resistance prediction for Mycobacterium tuberculosis from genome sequence data with Mykrobe. Wellcome Open Research, 2019. 4(191).

19. Walker, T.M., et al., Assessment of Mycobacterium tuberculosis transmission in Oxfordshire, UK, 2007-12, with whole pathogen genome sequences: an observational study. Lancet Respir Med, 2014. 2(4): p. 285-292.

20. Howe, K., A. Bateman, and R. Durbin, QuickTree: building huge Neighbour-Joining trees of protein sequences. Bioinformatics, 2002. 18(11): p. 1546-7.

21. Yu, G., Using ggtree to Visualize Data on Tree-Like Structures. Curr Protoc Bioinformatics, 2020. 69(1): p. e96.

22. Gagneux, S., Ecology and evolution of Mycobacterium tuberculosis. Nat Rev Microbiol, 2018. 16(4): p. 202-213.

23. Coscolla, M. and S. Gagneux, Consequences of genomic diversity in Mycobacterium tuberculosis. Semin Immunol, 2014. 26(6): p. 431-44.

24. Holt, K.E., et al., Frequent transmission of the Mycobacterium tuberculosis Beijing lineage and positive selection for the EsxW Beijing variant in Vietnam. Nat Genet, 2018. 50(6): p. 849-856.

25. Chiner-Oms, A. and I. Comas, Large genomics datasets shed light on the evolution of the Mycobacterium tuberculosis complex. Infect Genet Evol, 2019. 72: p. 10-15.

26. Freschi, L., et al., Population structure, biogeography and transmissibility of Mycobacterium tuberculosis. bioRxiv, 2020: p. 2020.09.29.293274.

27. Borrell, S., et al., Reference set of Mycobacterium tuberculosis clinical strains: A tool for research and product development. PLoS One, 2019. 14(3): p. e0214088.

28. Sharma, R., et al., High degree of fluoroquinolone resistance among pulmonary tuberculosis patients in New Delhi, India. Indian J Med Res, 2019. 149(1): p. 62-66.

29. Ghimire, S., et al., Treatment outcomes of patients with MDR-TB in Nepal on a current programmatic standardised regimen: retrospective single-centre study. BMJ Open Respir Res, 2020. 7(1). 
30. Oppong, Y.E.A., et al., Genome-wide analysis of Mycobacterium tuberculosis polymorphisms reveals lineage-specific associations with drug resistance. BMC Genomics, 2019. 20(1): p. 252.

31. Ford, C.B., et al., Mycobacterium tuberculosis mutation rate estimates from different lineages predict substantial differences in the emergence of drug-resistant tuberculosis. Nat Genet, 2013. 45(7): p. 784-90.

32. Berrada, Z.L., et al., Rifabutin and rifampin resistance levels and associated rpoB mutations in clinical isolates of Mycobacterium tuberculosis complex. Diagn Microbiol Infect Dis, 2016. 85(2): p. 177-81.

33. Maitre, T., et al., Are moxifloxacin and levofloxacin equally effective to treat XDR tuberculosis? J Antimicrob Chemother, 2017. 72(8): p. 2326-2333.

34. Dijkstra, J.A., et al., In Vitro Susceptibility of Mycobacterium tuberculosis to Amikacin, Kanamycin, and Capreomycin. Antimicrob Agents Chemother, 2018. 62(3).

35. Ho, J., P. Jelfs, and V. Sintchenko, Fluoroquinolone resistance in non-multidrugresistant tuberculosis-a surveillance study in New South Wales, Australia, and a review of global resistance rates. Int J Infect Dis, 2014. 26: p. 149-53.

36. Miotto, P., et al., A standardised method for interpreting the association between mutations and phenotypic drug resistance in Mycobacterium tuberculosis. Eur Respir J, 2017. 50(6).

37. Timothy M Walker, P.M., Claudio Köser†, Philip Fowler, Jeff Knaggs, Zamin Iqbal, Martin Hunt, Leonid Chindelevitch, Maha Farhat, Daniela Cirillo, Inaki Comas, Jamie Posey, Shaheed Vally Omar, Tim Peto, Anita Suresh, Swapna Uplekar, Sacha Laurant, Rebecca Colman, Carl-Michael Nathanson, Matteo Zignol, Ann Sarah Walker, The CRyPTIC Consortium, The Seq\&Treat Consortium, Derrick Crook, Nazir Ismail and Timothy Rodwell, The 2021 WHO catalogue of Mycobacterium tuberculosis mutations associated with drug resistance: a new global standard for molecular diagnostics (in prep). 2021.

38. Comas, I., et al., Whole-genome sequencing of rifampicin-resistant Mycobacterium tuberculosis strains identifies compensatory mutations in RNA polymerase genes. Nat Genet, 2011. 44(1): p. 106-10.

39. Almeida, D., et al., Mutations in pepQ Confer Low-Level Resistance to Bedaquiline and Clofazimine in Mycobacterium tuberculosis. Antimicrob Agents Chemother, 2016. 60(8): p. 4590-9.

40. Degiacomi, G., et al., In vitro Study of Bedaquiline Resistance in Mycobacterium tuberculosis Multi-Drug Resistant Clinical Isolates. Front Microbiol, 2020. 11: p. 559469.

41. Hartkoorn, R.C., S. Uplekar, and S.T. Cole, Cross-resistance between clofazimine and bedaquiline through upregulation of MmpL5 in Mycobacterium tuberculosis.

Antimicrob Agents Chemother, 2014. 58(5): p. 2979-81.

42. Nimmo, C., et al., Population-level emergence of bedaquiline and clofazimine resistance-associated variants among patients with drug-resistant tuberculosis in southern Africa: a phenotypic and phylogenetic analysis. Lancet Microbe, 2020. 1(4): p. e165-e174.

43. Singh, R., et al., PA-824 kills nonreplicating Mycobacterium tuberculosis by intracellular NO release. Science, 2008. 322(5906): p. 1392-5.

44. Mvelase, N.R., et al., Evolving rifampicin and isoniazid mono-resistance in a high multidrug-resistant and extensively drug-resistant tuberculosis region: a retrospective data analysis. BMJ Open, 2019. 9(11): p. e031663. 
1177

1178

1179

1180

1181

1182

1183

1184

1185

1186

1187

1188

1189

1190

1191

1192

1193

1194

1195

1196

1197

1198

1199

1200

1201

1202

1203

1204

1205

1206

1207

1208

1209

1210

1211

1212

1213

1214

1215

1216

1217

1218

1219

1220

1221

1222

45. Park, S., et al., Treatment outcomes of rifampin-sparing treatment in patients with pulmonary tuberculosis with rifampin-mono-resistance or rifampin adverse events: A retrospective cohort analysis. Respir Med, 2017. 131: p. 43-48.

46. Villegas, L., et al., Prevalence, Risk Factors, and Treatment Outcomes of Isoniazidand Rifampicin-Mono-Resistant Pulmonary Tuberculosis in Lima, Peru. PLoS One, 2016. 11(4): p. e0152933.

47. Oppong, Y.E.A., et al., Genome-wide analysis of Mycobacterium tuberculosis polymorphisms reveals lineage-specific associations with drug resistance. BMC Genomics, 2019. 20(1): p. 252.

48. Pang, Y., et al., Study of the rifampin monoresistance mechanism in Mycobacterium tuberculosis. Antimicrob Agents Chemother, 2013. 57(2): p. 893-900.

49. Kigozi, E., et al., Prevalence and patterns of rifampicin and isoniazid resistance conferring mutations in Mycobacterium tuberculosis isolates from Uganda. PLoS One, 2018. 13(5): p. e0198091.

50. Dean, A.S., et al., Prevalence and genetic profiles of isoniazid resistance in tuberculosis patients: A multicountry analysis of cross-sectional data. PLoS Med, 2020. 17(1): p. e1003008.

51. Migliori, G.B., et al., Protecting the tuberculosis drug pipeline: stating the case for the rational use of fluoroquinolones. Eur Respir J, 2012. 40(4): p. 814-22.

52. Heysell, S.K., et al., Hearing loss with kanamycin treatment for multidrug-resistant tuberculosis in Bangladesh. Eur Respir J, 2018. 51(3).

53. Organisation, W.H., Update on the use of nucleic acid amplification tests to detect TB and drug-resistant TB: rapid communication. 2021.

54. Olaru, I.D., et al., Bedaquiline-based treatment regimen for multidrug-resistant tuberculosis. Eur Respir J, 2017. 49(5).

55. Yuan, S., et al., Clofazimine broadly inhibits coronaviruses including SARS-CoV-2. Nature, 2021. 593(7859): p. 418-423.

56. Falzon, D., et al., World Health Organization treatment guidelines for drug-resistant tuberculosis, 2016 update. Eur Respir J, 2017. 49(3).

57. Huitric, E., et al., Rates and mechanisms of resistance development in Mycobacterium tuberculosis to a novel diarylquinoline ATP synthase inhibitor. Antimicrob Agents Chemother, 2010. 54(3): p. 1022-8.

58. Carter J, The CRyPTIC Consortium, Quantitative measurement of antibiotic resistance in Mycobacterium tuberculosis reveals genetic determinants of resistance and susceptibility in a target gene approach. 2021, in preparation.

59. Earle S and Wilson D, The CRyPTIC Consortium, Genome-wide association studies of global Mycobacterium tuberculosis resistance to thirteen antimicrobials in 10,228 genomes. 2021, in preparation.

60. Lachapelle AS, The CRyPTIC Consortium, Predicting Susceptibility to First- and Second-line Tuberculosis Drugs by DNA sequencing and Machine Learning. 2021, in preparation.

61. Sonnenkalb, L., et al., Deciphering Bedaquiline and Clofazimine Resistance in Tuberculosis: An Evolutionary Medicine Approach. bioRxiv, 2021: p. 2021.03.19.436148. 